\title{
A Tutorial on the Basic Special Functions of Fractional Calculus
}

\author{
FRANCESCO MAINARDI \\ University Bologna and INFN \\ Department of Physics \& Astronomy
}

ITALY

\begin{abstract}
In this tutorial survey we recall the basic properties of the special function of the Mittag-Leffler and Wright type that are known to be relevant in processes dealt with the fractional calculus. We outline the major applications of these functions. For the Mittag-Leffler functions we analyze the Abel integral equation of the second kind and the fractional relaxation and oscillation phenomena. For the Wright functions we distinguish them in two kinds. We mainly stress the relevance of the Wright functions of the second kind in probability theory with particular regard to the so-called $M$-Wright functions that generalizes the Gaussian and is related with the time-fractional diffusion equation.
\end{abstract}

Key-Words: Mittag-Leffler functions, Wright functions, Fractional Calculus, Laplace, Fourier and Mellin transforms, Probability theory, Stable distributions.

Received: November 18, 2019. Revised: March 9, 2020. Accepted: April 14, 2020. Published: April 24, 2020.

\section{Introduction}

The special functions of the Mittag-Leffer and Wright type in general play a very important role in the theory of the fractional differential and integral equations. The purpose of this tutorial survey is to outline the relevant properties of the these functions outlining their applications.

This work is organized as follows. In Section 2, we recall the essentials of the fractional calculus that provide the necessary notions for the applications.

In section 3, we start to define the Mittag-Leffler functions. For this purpose we introduce the Gamma function and the classical Mittag-Leffler functions of one and two parameters. Then we deal with the auxiliary functions of the Mittag-Leffler type to be used in the next sections.

In Section 4 we apply the above auxiliary functions of the Mittag-Leffler type to solve the Abel integral equations of the second kind, that are noteworthy cases of Volterra integral equations.

In Section 5 we finally consider the most famous applications of the auxiliary functions of the MittagLeffler tyewpe, that is the solutions of the time fractional differential equations governing the phenomena of fractional relaxation and fractional oscillations In Section 6 we start to define the Wright functions. For this purpose we distinguish two kinds of these functions. Particular attention is devoted to two special cases of the Wright function of the second kind introduced by Mainardi in the 1990's in virtue of their importance in probability theory and for the time- fractional diffusion equations. Nowadays in the FC literature they are referred to as the Mainardi functions. In contrast to the general case of the Wright function, they depend just on one parameter $\nu \in$ $[0,1)$. One of the Mainardi functions, known as the $M$-Wright function, generalizes the Gaussian function and degenerates to the delta function in the limiting case $\nu=1$.

Then, in Section 7 we recall how the Mainardi functions are related to an important class of the probability density functions (pdf's) known as the extremal Lévy stable densities. This emphasizes the relevance of the Mainardi functions in the probability theory independently on the framework of the fractional diffusion equations. We present some plots of the symmetric $M$-Wright function on $\mathbb{R}$ for several parameter values $\nu \in[0,1 / 2]$ and $\nu \in[1 / 2,1]$.

Finally concluding remarks are carried out in Section 8 and two tutorial appendices on stable distributions and the time fractional diffusion equation are added for readers' convenience.

The paper is competed with historical and bibliographical concerning the past approach of the author towards the Wright functions.

\section{The essentials of fractional calculus}

This section is mainly based on the 1997 CISM survey by Gorenflo and Mainardi [19]. 
The Riemann-Liouville fractional integral of order $\mu>0$ is defined as

$$
{ }_{t} J^{\mu} f(t):=\frac{1}{\Gamma(\mu)} \int_{0}^{t}(t-\tau)^{\mu-1} f(\tau) d \tau,
$$

where

$$
\Gamma(\mu):=\int_{0}^{\infty} \mathrm{e}^{-u} u^{\mu-1} d u, \Gamma(n+1)=n !
$$

is theGamma function.

By convention ${ }_{t} J^{0}=I$ (Identity operator). We can prove semi-group property

$$
{ }_{t} J^{\mu}{ }_{t} J^{\nu}={ }_{t} J^{\nu}{ }_{t} J^{\mu}={ }_{t} J^{\mu+\nu}, \mu, \nu \geq 0 .
$$

Furthermore we have for $t>0$

$$
{ }_{t} J^{\mu} t^{\gamma}=\frac{\Gamma(\gamma+1)}{\Gamma(\gamma+1+\mu)} t^{\gamma+\mu}, \mu \geq 0, \gamma>-1 .
$$

The fractional derivative of order $\mu>0$ in the Riemann-Liouville sense is defined as the operator ${ }_{t} D^{\mu}$

$$
{ }_{t} D^{\mu}{ }_{t} J^{\mu}=I, \mu>0 .
$$

If we take $m-1<\mu \leq m$, with $m \in \mathbb{N}$ we recognize from Eqs. (2.2) and (2.4)

$$
{ }_{t} D^{\mu} f(t):={ }_{t} D^{m}{ }_{t} J^{m-\mu} f(t),
$$

hence, for $m-1<\mu<m$,

$$
{ }_{t} D^{\mu} f(t)=\frac{d^{m}}{d t^{m}}\left[\frac{1}{\Gamma(m-\mu)} \int_{0}^{t} \frac{f(\tau) d \tau}{(t-\tau)^{\mu+1-m}}\right],
$$

and, for $\mu=m$,

$$
{ }_{t} D^{\mu} f(t)=\frac{d^{m}}{d t^{m}} f(t), .
$$

For completion ${ }_{t} D^{0}=I$. The semi-group property is no longer valid but for $t>0$

$$
{ }_{t} D^{\mu} t^{\gamma}=\frac{\Gamma(\gamma+1)}{\Gamma(\gamma+1-\mu)} t^{\gamma-\mu}, \mu \geq 0, \gamma>-1 .
$$

However, the property ${ }_{t} D^{\mu}={ }_{t} J^{-\mu}$ is not generally valid!

The fractional derivative of order $\mu \in(m-1, m]$ $(m \in \mathbb{N})$ in the Caputo sense is defined as the operator ${ }_{t} D_{*}^{\mu}$ such that,

$$
{ }_{t} D_{*}^{\mu} f(t):={ }_{t} J^{m-\mu}{ }_{t} D^{m} f(t),
$$

hence, for $\mathrm{f} m-1<\mu<m$,

$$
{ }_{t} D_{*}^{\mu} f(t)=\frac{1}{\Gamma(m-\mu)} \int_{0}^{t} \frac{f^{(m)}(\tau) d \tau}{(t-\tau)^{\mu+1-m}},
$$

and for $\mu=m$

$$
{ }_{t} D_{*}^{m} f(t)=: \frac{d^{m}}{d t^{m}} f(t) .
$$

Thus, when the order is not integer the two fractional derivatives differ in that the derivative of order $m$ does not generally commute with the fractional integral.

We point out that the Caputo fractional derivative satisfies the relevant property of being zero when applied to a constant, and, in general, to any power function of non-negative integer degree less than $m$, if its order $\mu$ is such that $m-1<\mu \leq m$.

Gorenflo and Mainardi (1997) [19] have shown the essential relationships between the two fractional derivatives (when both of them exist), for $m-1<$ $\mu<m$,

$$
{ }_{t} D_{*}^{\mu} f(t)=\left\{\begin{array}{l}
{ }_{t} D^{\mu}\left[f(t)-\sum_{k=0}^{m-1} f^{(k)}\left(0^{+}\right) \frac{t^{k}}{k !}\right] \\
{ }_{t} D^{\mu} f(t)-\sum_{k=0}^{m-1} \frac{f^{(k)}\left(0^{+}\right) t^{k-\mu}}{\Gamma(k-\mu+1)}
\end{array}\right.
$$

In particular, if $m=1$ so that $0<\mu<1$, we have

$$
{ }_{t} D_{*}^{\mu} f(t)=\left\{\begin{array}{l}
{ }_{t} D^{\mu}\left[f(t)-f\left(0^{+}\right)\right], \\
{ }_{t} D^{\mu} f(t)-\frac{f\left(0^{+}\right) t^{-\mu}}{\Gamma(1-\mu)} .
\end{array}\right.
$$

The Caputo fractional derivative, represents a sort of regularization in the time origin for the RiemannLiouville fractional derivative.

We note that for its existence all the limiting values $f^{(k)}\left(0^{+}\right):=\lim _{t \rightarrow 0^{+}} f^{(k)}(t)$ are required to be finite for $k=0,1,2 \ldots m-1$.

We observe the different behaviour of the two fractional derivatives at the end points of the interval $(m-1, m)$ namely when the order is any positive integer: whereas ${ }_{t} D^{\mu}$ is, with respect to its order $\mu$, an operator continuous at any positive integer, ${ }_{t} D_{*}^{\mu}$ is an operator left-continuous since

$$
\begin{aligned}
& \lim _{\mu \rightarrow(m-1)^{+}}{ }_{t} D_{*}^{\mu} f(t)=f^{(m-1)}(t)-f^{(m-1)}\left(0^{+}\right), \\
& \lim _{\mu \rightarrow m^{-}}{ }_{t} D_{*}^{\mu} f(t)=f^{(m)}(t) .
\end{aligned}
$$


We also note for $m-1<\mu \leq m$,

${ }_{t} D^{\mu} f(t)={ }_{t} D^{\mu} g(t) \Longleftrightarrow f(t)=g(t)+\sum_{j=1}^{m} c_{j} t^{\mu-j}$,

${ }_{t} D_{*}^{\mu} f(t)={ }_{t} D_{*}^{\mu} g(t) \Longleftrightarrow f(t)=g(t)+\sum_{j=1}^{m} c_{j} t^{m-j}$.

In these formulae the coefficients $c_{j}$ are arbitrary constants.

We point out the major utility of the Caputo fractional derivative in treating initial-value problems for physical and engineering applications where initial conditions are usually expressed in terms of integer-order derivatives. This can be easily seen using the Laplace transformation.

Writing the Laplace transform of a sufficiently wellbehaved function $f(t)(t \geq 0)$ as

$$
\mathcal{L}\{f(t) ; s\}=\tilde{f}(s):=\int_{0}^{\infty} \mathrm{e}^{-s t} f(t) d t,
$$

the known rule for the ordinary derivative of integer order $m \in \mathbb{N}$ is

$\mathcal{L}\left\{{ }_{t} D^{m} f(t) ; s\right\}=s^{m} \widetilde{f}(s)-\sum_{k=0}^{m-1} s^{m-1-k} f^{(k)}\left(0^{+}\right)$,

where

$$
f^{(k)}\left(0^{+}\right):=\lim _{t \rightarrow 0^{+}}{ }_{t} D^{k} f(t) .
$$

For the Caputo derivative of order $\mu \in(m-1, m]$ $(m \in \mathbb{N})$ we have

$$
\begin{aligned}
& \mathcal{L}\left\{{ }_{t} D_{*}^{\mu} f(t) ; s\right\}=s^{\mu} \tilde{f}(s)-\sum_{k=0}^{m-1} s^{\mu-1-k} f^{(k)}\left(0^{+}\right), \\
& f^{(k)}\left(0^{+}\right):=\lim _{t \rightarrow 0^{+}}{ }_{t} D^{k} f(t) .
\end{aligned}
$$

The corresponding rule for the Riemann-Liouvile derivative of order $\mu$ is

$$
\begin{aligned}
& \mathcal{L}\left\{{ }_{t} D_{t}^{\mu} f(t) ; s\right\}=s^{\mu} \tilde{f}(s)-\sum_{k=0}^{m-1} s^{m-1-k} g^{(k)}\left(0^{+}\right), \\
& g^{(k)}\left(0^{+}\right):=\lim _{t \rightarrow 0^{+}}{ }_{t} D^{k} g(t), g(t):={ }_{t} J^{m-\mu} f(t) .
\end{aligned}
$$

Thus the rule (2.14) is more cumbersome to be used than (2.13) since it requires initial values concerning an extra function $g(t)$ related to the given $f(t)$ through a fractional integral.

However, when all the limiting values $f^{(k)}\left(0^{+}\right)$are finite and the order is not integer, we can prove by that all $g^{(k)}\left(0^{+}\right)$vanish so that the formula (2.14) simplifies into

$$
\mathcal{L}\left\{{ }_{t} D^{\mu} f(t) ; s\right\}=s^{\mu} \tilde{f}(s), m-1<\mu<m .
$$

For this proof it is sufficient to apply the Laplace transform to Eq. (2.8), by recalling that

$$
\mathcal{L}\left\{t^{\nu} ; s\right\}=\Gamma(\nu+1) / s^{\nu+1}, \quad \nu>-1,
$$

and then to compare (2.13) with (2.14).

\section{Ihe function of Mittag-Leffler type}

We note that the Mittag-Leffler functions are present in the Mathematics Subject Classification since the year 2000 under the number 33E12 under recommendation of Prof. Gorenflo.

A description of the most important properties of these functions (with relevant references up to the fifties) can be found in the third volume of the Bateman Project edited by Erdelyi et al. (1955) in the chapter XVIII on Miscellaneous Functions [10].

The treatises where great attention is devoted to the functions of the Mittag-Leffler type is that by Djrbashian (1966) [9], unfortunately in Russian.

We also recommend the classical treatise on complex functions by Sansone \& Gerretsen (1960) [54].

Nowadays the Mittag-Leffler functions are widely used in the framework of integral and differential equations of fractional order, as shown in all treatises on fractional calculus.

In view of its several applications in Fractional Calculus the Mittag-Leffler function was referred to as the Queen Function of Fractional Calculus by Mainardi \& Gorenflo (2007) [34].

Finally, the functions of the Mittag-Leffler type have found an exhaustive treatment in the treatise by Gorenflo, Kilbas, Mainardi \& Rogosin (2014) [15]

As pioneering works of mathematical nature in the field of fractional integral and differential equations, we like to quote Hille \& Tamarkin (1930) [22] who have provided the solution of the Abel integral equation of the second kind in terms of a Mittag-Leffler function, and Barret (1954) [1] who has expressed the general solution of the linear fractional differential equation with constant coefficients in terms of MittagLeffler functions.

As former applications in physics we like to quote the contributions by Cole (1933) [5] in connection with 
nerve conduction, see also Davis (1936) [7],and by Gross (1947) [21] in connection with mechanical relaxation.

Subsequently, Caputo \& Mainardi (1971a), (1971b) $[3,4]$ have shown that Mittag-Leffler functions are present whenever derivatives of fractional order are introduced in the constitutive equations of a linear viscoelastic body. Since then, several other authors have pointed out the relevance of these functions for fractional viscoelastic models, see e.g. Mainardi's survey (1997) [31] and his (2010) book [32].

\subsection{The Gamma function: $\Gamma(z)$}

The Gamma function $\Gamma(z)$ is the most widely used of all the special functions: it is usually discussed first because it appears in almost every integral or series representation of other advanced mathematical functions. The first occurrence of the gamma function happens in 1729 in a correspondence between $\mathrm{Eu}-$ ler and Goldbach. We take as its definition the integral formula due to Legendre (1809)

$$
\Gamma(z):=\int_{0}^{\infty} u^{z-1} \mathrm{e}^{-u} d u, \quad \mathcal{R} e(z)>0 .
$$

This integral representation is the most common for $\Gamma(z)$, even if it is valid only in the right half-plane of $\mathbb{C}$.

The analytic continuation to the left half-plane is possible in different ways. As will be shown hereafter, the domain of analyticity $D_{\Gamma}$ of $\Gamma(z)$ turns out to be

$$
D_{\Gamma}=\mathbb{C}-\{0,-1,-2, \ldots\} .
$$

The most common continuation is carried out by the mixed representation due to Mittag-Leffler: and reads for $z \in D_{\Gamma}$

$$
\Gamma(z)=\sum_{n=0}^{\infty} \frac{(-1)^{n}}{n !(z+n)}+\int_{1}^{\infty} e^{-u} u^{z-1} d u .
$$

This representation can be obtained from the so-called Prym's decomposition, namely by splitting the integral in (3.1) into 2 integrals, the one over the interval $0 \leq u \leq 1$ which is then developed in a series, the other over the interval $1 \leq u \leq \infty$, which, being uniformly convergent inside $\mathbb{C}$, provides an entire function. The terms of the series (uniformly convergent inside $\left.D_{\Gamma}\right)$ provide the principal parts of $\Gamma(z)$ at the corresponding poles $z_{n}=-n$. So we recognize that $\Gamma(z)$ is analytic in the entire complex plane except at the points $z_{n}=-n(n=0,1, \ldots)$, which turn out to be simple poles with residues $R_{n}=(-1)^{n} / n$ !. The point at infinity, being an accumulation point of poles, is an essential non-isolated singularity. Thus $\Gamma(z)$ is a transcendental meromorphic function.

The reciprocal of the Gamma function turns out to be an entire function. its integral representation in the complex plane was due to Hankel (1864) and reads

$$
\frac{1}{\Gamma(z)}=\frac{1}{2 \pi i} \int_{H a} \frac{\mathrm{e}^{u}}{u^{z}} d u, z \in \mathbb{C},
$$

where $H a$ denotes the Hankel path defined as a contour that begins at $u=-\infty-i a(a>0)$, encircles the branch cut that lies along the negative real axis, and ends up at $u=-\infty+i b(b>0)$. Of course, the branch cut is present when $z$ is non-integer because $u^{-z}$ is a multivalued function; when $z$ is an integer, the contour can be taken to be simply a circle around the origin, described in the counterclockwise direction.

\subsection{The classical Mittag-Leffler functions}

The Mittag-Leffler functions, that we denote by $E_{\alpha}(z), E_{\alpha, \beta}(z)$ are so named in honour of Gösta Mittag-Leffler, the eminent Swedish mathematician, who introduced and investigated these functions in a series of notes starting from 1903 in the framework of the theory of entire functions [44, 45, 46, 47]. The functions are defined by the series representations, convergent in the whole complex plane $\mathbb{C}$ for $\operatorname{Re}(\alpha)>0\}$

$$
\left\{\begin{array}{l}
E_{\alpha}(z):=\sum_{n=0}^{\infty} \frac{z^{n}}{\Gamma(\alpha n+1)}, \\
E_{\alpha, \beta}(z):=\sum_{n=0}^{\infty} \frac{z^{n}}{\Gamma(\alpha n+\beta)},
\end{array}\right.
$$

with $\beta \in \mathbb{C}$..

Originally Mittag-Leffler assumed only the parameter $\alpha$ and assumed it as positive, but soon later the generalization with two complex parameters was considered by Wiman. [59]. In both cases the Mittag-Leffler functions are entire of order $1 / \operatorname{Re}(\alpha)$. The integral representation for $z \in \mathbb{C}$ introduced by Mittag-Leffler can be written as

$$
E_{\alpha}(z)=\frac{1}{2 \pi i} \int_{H a} \frac{\zeta^{\alpha-1} \mathrm{e}^{\zeta}}{\zeta^{\alpha}-z} d \zeta, \alpha>0 .
$$

Using series representations of the Mittag-Leffler 
functions it is easy to recognize

$\left\{\begin{array}{l}E_{1,1}(z)=E_{1}(z)=\mathrm{e}^{z}, E_{1,2}(z)=\frac{\mathrm{e}^{z}-1}{z}, \\ E_{2,1}\left(z^{2}\right)=\cosh (z), E_{2,1}\left(-z^{2}\right)=\cos (z), \\ E_{2,2}\left(z^{2}\right)=\frac{\sinh (z)}{z}, E_{2,2}\left(-z^{2}\right)=\frac{\sin (z)}{z},\end{array}\right.$

and more generally

$$
\left\{\begin{array}{l}
E_{\alpha, \beta}(z)+E_{\alpha, \beta}(-z)=2 E_{2 \alpha, \beta}\left(z^{2}\right), \\
E_{\alpha, \beta}(z)-E_{\alpha, \beta}(-z)=2 z E_{2 \alpha, \alpha+\beta}\left(z^{2}\right) .
\end{array}\right.
$$

Furthermore, for $\alpha=1 / 2$,

$$
\begin{aligned}
E_{1 / 2}\left( \pm z^{1 / 2}\right) & =\mathrm{e}^{z}\left[1+\operatorname{erf}\left( \pm z^{1 / 2}\right)\right] \\
& =\mathrm{e}^{z} \operatorname{erfc}\left(\mp z^{1 / 2}\right),
\end{aligned}
$$

where erf (erfc) denotes the (complementary) error function defined for $z \in \mathbb{C}$ as

$$
\operatorname{erf}(z):=\frac{2}{\sqrt{\pi}} \int_{0}^{z} \mathrm{e}^{-u^{2}} d u, \operatorname{erfc}(z):=1-\operatorname{erf}(z) .
$$

\subsection{The auxiliary functions of the Mittag- Leffler type}

In view of applications we introduce the following causal functions in time domain

$$
\begin{aligned}
& e_{\alpha}(t ; \lambda):=E_{\alpha}\left(-\lambda t^{\alpha}\right) \div \frac{s^{\alpha-1}}{s^{\alpha}+\lambda} \\
& e_{\alpha, \beta}(t ; \lambda):=t^{\beta-1} E_{\alpha, \beta}\left(-\lambda t^{\alpha}\right) \div \frac{s^{\alpha-\beta}}{s^{\alpha}+\lambda} \\
& e_{\alpha, \alpha}(t ; \lambda):=t^{\alpha-1} E_{\alpha, \alpha}\left(-\lambda t^{\alpha}\right) \\
&=\frac{d}{d t} e_{\alpha}\left(-\lambda t^{\alpha}\right) \div-\frac{\lambda}{s^{\alpha}+\lambda} .
\end{aligned}
$$

A function $f(t)$ defined in $\mathbb{R}^{+}$is completely monotone $(\mathrm{CM})$ if $(-1)^{n} f^{n}(t) \geq 0$. The function $\mathrm{e}^{-t}$ is the prototype of a $\mathrm{CM}$ function.

For a Bernstein theorem a generic $\mathrm{CM}$ function reads

$$
f(t)=\int_{0}^{\infty} \mathrm{e}^{-r t} K(r) \mathrm{d} r, K(r) \geq 0 .
$$

We have for $\lambda>0$

$$
\begin{aligned}
& e_{\alpha, \beta}(t ; \lambda):=t^{\beta-1} E_{\alpha, \beta}\left(-\lambda t^{\alpha}\right) \\
& \mathrm{CM} \text { iff } 0<\alpha \leq \beta \leq 1 .
\end{aligned}
$$

Using the Laplace transform we can prove, following Gorenflo and Mainardi (1997) [19] that for $0<\alpha<1$ (with $\lambda=1$ )

$E_{\alpha}\left(-t^{\alpha}\right) \simeq \begin{cases}1-\frac{t^{\alpha}}{\Gamma(\alpha+1)} \cdots & t \rightarrow 0^{+}, \\ \frac{t^{-\alpha}}{\Gamma(1-\alpha)} \cdots & t \rightarrow+\infty,\end{cases}$

and

$$
E_{\alpha}\left(-t^{\alpha}\right)=\int_{0}^{\infty} \mathrm{e}^{-r t} K_{\alpha}(r) \mathrm{d} r
$$

with

$K_{\alpha}(r)=\frac{1}{\pi} \frac{r^{\alpha-1} \sin (\alpha \pi)}{r^{2 \alpha}+2 r^{\alpha} \cos (\alpha \pi)+1}>0$.

In the following sections we will outline the key role of the auxiliary functions in the treatment of integral and differential equations of fractional order, including the Abel integral equation of the second kind and the differential equations for fractional relaxation and oscillation.

Before closing this section we find it convenient to provide the plots of the functions

$$
\psi_{\alpha}(t)=e_{\alpha}(t):=E_{\alpha}\left(-t^{\alpha}\right),
$$

and

$$
\phi_{\alpha}(t)=t^{-(1-\alpha)} E_{\alpha, \alpha}\left(-t^{\alpha}\right):=-\frac{d}{d t} E_{\alpha}\left(-t^{\alpha}\right),
$$

for $t \geq 0$ and for some rational values of $\alpha \in(0,1]$.

For the sake of visibility, for both functions we have adopted linear and logarithmic scales. Logarithmic scales have been adopted in order to better point out their asymptotic behaviour for large times.

It is worth noting the algebraic decay of $\psi_{\alpha}(t)$ and $\phi_{\alpha}(t)$

$$
\begin{aligned}
& \psi_{\alpha}(t) \sim \frac{\sin (\alpha \pi)}{\pi} \frac{\Gamma(\alpha)}{t^{\alpha}} \\
& \phi_{\alpha}(t) \sim \frac{\sin (\alpha \pi)}{\pi} \frac{\Gamma(\alpha+1)}{t^{(\alpha+1)}},
\end{aligned}
$$

\section{Abel integral equation of the second kind}

Let us now consider the Abel equation of the second kind with $\alpha>0, \lambda \in \mathbb{C}$ :

$$
u(t)+\frac{\lambda}{\Gamma(\alpha)} \int_{0}^{t} \frac{u(\tau)}{(t-\tau)^{1-\alpha}} d \tau=f(t)
$$



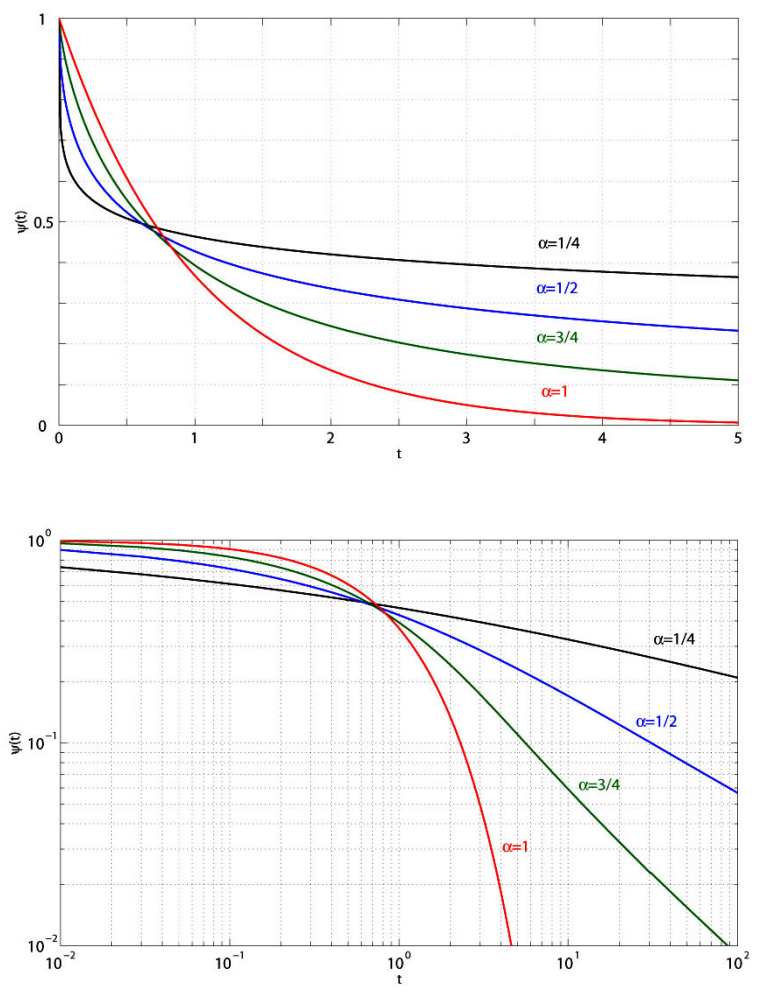

Figure 1: Plots of $\psi_{\alpha}(t)$ with $\alpha=1 / 4,1 / 2,3 / 4,1$ top: linear scales; bottom: logarithmic scales.
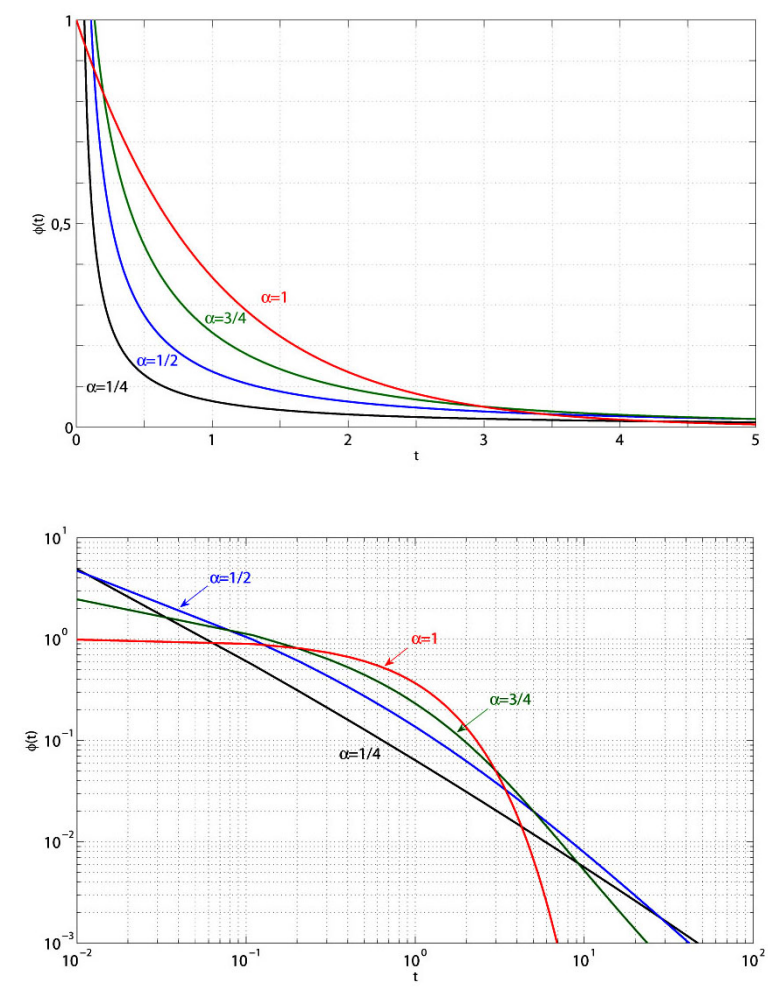

Figure 2: Plots of $\phi_{\alpha}(t)$ with $\alpha=1 / 4,1 / 2,3 / 4,1$ top: linear scales; bottom: logarithmic scales.
In terms of the fractional integral operator such equation reads

$$
\left(1+\lambda J^{\alpha}\right) u(t)=f(t)
$$

and consequently can be formally solved as follows:

$$
\begin{aligned}
u(t) & =\left(1+\lambda J^{\alpha}\right)^{-1} f(t) \\
& =\left(1+\sum_{n=1}^{\infty}(-\lambda)^{n} J^{\alpha n}\right) f(t) .
\end{aligned}
$$

Recalling the definition of the fractional integral the formal solution reads

$$
u(t)=f(t)+\left(\sum_{n=1}^{\infty}(-\lambda)^{n} \frac{t_{+}^{\alpha n-1}}{\Gamma(\alpha n)}\right) * f(t) .
$$

Recalling the definition of the function,

$$
e_{\alpha}(t ; \lambda):=E_{\alpha}\left(-\lambda t^{\alpha}\right)=\sum_{n=0}^{\infty} \frac{\left(-\lambda t^{\alpha}\right)^{n}}{\Gamma(\alpha n+1)},
$$

where $E_{\alpha}$ denotes the Mittag-Leffler function of order $\alpha$, we note that for $t>0$ :

$$
\sum_{n=1}^{\infty}(-\lambda)^{n} \frac{t_{+}^{\alpha n-1}}{\Gamma(\alpha n)}=\frac{d}{d t} E_{\alpha}\left(-\lambda t^{\alpha}\right)=e_{\alpha}^{\prime}(t ; \lambda),
$$

Finally, the solution reads

$$
u(t)=f(t)+e_{\alpha}^{\prime}(t ; \lambda) * f(t) .
$$

Of course the above formal proof can be made rigorous. Simply observe that because of the rapid growth of the gamma function the infinite series in (4.4) and (4.6) are uniformly convergent in every bounded interval of the variable $t$ so that term-wise integrations and differentiations are allowed.

However, we prefer to use the alternative technique of Laplace transforms, which will allow us to obtain the solution in different forms, including the result (4.7).

Applying the Laplace transform to (4.1) we obtain

$$
\left[1+\frac{\lambda}{s^{\alpha}}\right] \bar{u}(s)=\bar{f}(s) \Longrightarrow \bar{u}(s)=\frac{s^{\alpha}}{s^{\alpha}+\lambda} \bar{f}(s) .
$$

Now, let us proceed to obtain the inverse Laplace transform of (4.8) using the following Laplace transform pair

$$
e_{\alpha}(t ; \lambda):=E_{\alpha}\left(-\lambda t^{\alpha}\right) \div \frac{s^{\alpha-1}}{s^{\alpha}+\lambda} .
$$


We can choose three different ways to get the inverse Laplace transforms from (4.8), according to the standard rules. Writing (4.8) as

$$
\bar{u}(s)=s\left[\frac{s^{\alpha-1}}{s^{\alpha}+\lambda} \bar{f}(s)\right],
$$

we obtain

$$
u(t)=\frac{d}{d t} \int_{0}^{t} f(t-\tau) e_{\alpha}(\tau ; \lambda) d \tau .
$$

If we write (4.8) as

$$
\bar{u}(s)=\frac{s^{\alpha-1}}{s^{\alpha}+\lambda}\left[s \bar{f}(s)-f\left(0^{+}\right)\right]+f\left(0^{+}\right) \frac{s^{\alpha-1}}{s^{\alpha}+\lambda},
$$

we obtain

$$
u(t)=\int_{0}^{t} f^{\prime}(t-\tau) e_{\alpha}(\tau ; \lambda) d \tau+f\left(0^{+}\right) e_{\alpha}(t ; \lambda) .
$$

We also note that, $e_{\alpha}(t ; \lambda)$ being a function differentiable with respect to $t$ with

$$
e_{\alpha}\left(0^{+} ; \lambda\right)=E_{\alpha}\left(0^{+}\right)=1,
$$

there exists another possibility to re-write (4.8), namely

$$
\bar{u}(s)=\left[s \frac{s^{\alpha-1}}{s^{\alpha}+\lambda}-1\right] \bar{f}(s)+\bar{f}(s) .
$$

Then we obtain

$$
u(t)=\int_{0}^{t} f(t-\tau) e_{\alpha}^{\prime}(\tau ; \lambda) d \tau+f(t),
$$

in agreement with (4.7). We see that the way $b$ ) is more restrictive than the ways $a$ ) and $c$ ) since it requires that $f(t)$ be differentiable with $\mathcal{L}$ transformable derivative.

\section{Fractional relaxation and oscillations}

Generally speaking, we consider the following differential equation of fractional order $\alpha>0$, for $t \geq 0$ :

$$
\begin{aligned}
D_{*}^{\alpha} u(t) & =D^{\alpha}\left(u(t)-\sum_{k=0}^{m-1} \frac{t^{k}}{k !} u^{(k)}\left(0^{+}\right)\right) \\
& =-u(t)+q(t)
\end{aligned}
$$

where $u=u(t)$ is the field variable and $q(t)$ is a given function, continuous for $t \geq 0$. Here $m$ is a positive integer uniquely defined by $m-1<\alpha \leq m$, which provides the number of the prescribed initial values $u^{(k)}\left(0^{+}\right)=c_{k}, k=0,1,2, \ldots, m-1$.

In particular, we consider in detail the cases

(a) fractional relaxation $0<\alpha \leq 1$,

(b) fractional oscillation $1<\alpha \leq 2$.

The application of the Laplace transform yields

$$
\widetilde{u}(s)=\sum_{k=0}^{m-1} c_{k} \frac{s^{\alpha-k-1}}{s^{\alpha}+1}+\frac{1}{s^{\alpha}+1} \widetilde{q}(s) .
$$

Then, putting for $k=0,1, \ldots, m-1$,

$$
\begin{aligned}
& u_{k}(t):=J^{k} e_{\alpha}(t) \div \frac{s^{\alpha-k-1}}{s^{\alpha}+1}, \\
& e_{\alpha}(t):=E_{\alpha}\left(-t^{\alpha}\right) \div \frac{s^{\alpha-1}}{s^{\alpha}+1},
\end{aligned}
$$

and using $u_{0}\left(0^{+}\right)=1$, we find,

$$
u(t)=\sum_{k=0}^{m-1} c_{k} u_{k}(t)-\int_{0}^{t} q(t-\tau) u_{0}^{\prime}(\tau) d \tau .
$$

In particular, the formula (5.4) encompasses the solutions for $\alpha=1,2$, since

$$
\begin{gathered}
\alpha=1, u_{0}(t)=e_{1}(t)=\exp (-t) \\
\alpha=2, u_{0}(t)=e_{2}(t)=\cos t, u_{1}(t)=J^{1} e_{2}(t)=\sin t
\end{gathered}
$$

When $\alpha$ is not integer, namely for $m-1<\alpha<m$, we note that $m-1$ represents the integer part of $\alpha$ (usually denoted by $[\alpha]$ ) and $m$ the number of initial conditions necessary and sufficient to ensure the uniqueness of the solution $u(t)$. Thus the $m$ functions $u_{k}(t)=J^{k} e_{\alpha}(t)$ with $k=0,1, \ldots, m-1$ represent those particular solutions of the homogeneous equation which satisfy the initial conditions $u_{k}^{(h)}\left(0^{+}\right)=$ $\delta_{k h}, h, k=0,1, \ldots, m-1$, and therefore they represent the fundamental solutions of the fractional equation (5.1), in analogy with the case $\alpha=m$. Furthermore, the function $u_{\delta}(t)=-u_{0}^{\prime}(t)=-e_{\alpha}^{\prime}(t)$ represents the impulse-response solution.

Now we derive the relevant properties of the basic functions $e_{\alpha}(t)$ directly from their Laplace representation for $0<\alpha \leq 2$,

$$
e_{\alpha}(t)=\frac{1}{2 \pi i} \int_{B r} \mathrm{e}^{s t} \frac{s^{\alpha-1}}{s^{\alpha}+1} d s,
$$

without detouring on the general theory of MittagLeffler functions in the complex plane. Here $\mathrm{Br}$ denotes a Bromwich path, i.e. a line $\operatorname{Re}(s)=\sigma>0$ and $\operatorname{Im}(s)$ running from $-\infty$ to $+\infty$. 
For transparency reasons, we separately discuss the cases (a) $0<\alpha<1$ and (b) $1<\alpha<2$, recalling that in the limiting cases $\alpha=1,2$, we know $e_{\alpha}(t)$ as elementary function, namely $e_{1}(t)=\mathrm{e}^{-t}$ and $e_{2}(t)=\cos t$.

For $\alpha$ not integer the power function $s^{\alpha}$ is uniquely defined as $s^{\alpha}=|s|^{\alpha} \mathrm{e}^{i \arg s}$, with $-\pi<\arg s<\pi$, that is in the complex $s$-plane cut along the negative real axis.

The essential step consists in decomposing $e_{\alpha}(t)$ into two parts according to $e_{\alpha}(t)=f_{\alpha}(t)+g_{\alpha}(t)$, as indicated below. In case (a) the function $f_{\alpha}(t)$, in case (b) the function $-f_{\alpha}(t)$ is completely monotone; in both cases $f_{\alpha}(t)$ tends to zero as $t$ tends to infinity, from above in case (a), from below in case (b). The other part, $g_{\alpha}(t)$, is identically vanishing in case (a), but of oscillatory character with exponentially decreasing amplitude in case (b).

For the oscillatory part we obtain via the residue theorem of complex analysis, when $1<\alpha<2$ :

$$
g_{\alpha}(t)=\frac{2}{\alpha} \mathrm{e}^{t \cos (\pi / \alpha)} \cos \left[t \sin \left(\frac{\pi}{\alpha}\right)\right] .
$$

We note that this function exhibits oscillations with circular frequency

$$
\omega(\alpha)=\sin (\pi / \alpha)
$$

and with an exponentially decaying amplitude with rate

$$
\lambda(\alpha)=|\cos (\pi / \alpha)|=-\cos (\pi / \alpha) .
$$

For the monotonic part we obtain

$$
f_{\alpha}(t):=\int_{0}^{\infty} \mathrm{e}^{-r t} K_{\alpha}(r) d r
$$

with

$$
\begin{aligned}
K_{\alpha}(r) & =-\frac{1}{\pi} \operatorname{Im}\left(\left.\frac{s^{\alpha-1}}{s^{\alpha}+1}\right|_{s=r \mathrm{e}^{i \pi}}\right) \\
& =\frac{1}{\pi} \frac{r^{\alpha-1} \sin (\alpha \pi)}{r^{2 \alpha}+2 r^{\alpha} \cos (\alpha \pi)+1} .
\end{aligned}
$$

This function $K_{\alpha}(r)$ vanishes identically if $\alpha$ is an integer, it is positive for all $r$ if $0<\alpha<1$, negative for all $r$ if $1<\alpha<2$. In fact in (5.8) the denominator is, for $\alpha$ not integer, always positive being $>\left(r^{\alpha}-\right.$ $1)^{2} \geq 0$.

Hence $f_{\alpha}(t)$ has the aforementioned monotonicity properties, decreasing towards zero in case (a), increasing towards zero in case (b).
We note that, in order to satisfy the initial condition $e_{\alpha}\left(0^{+}\right)=1$, we find

$$
\begin{gathered}
\int_{0}^{\infty} K_{\alpha}(r) d r=1 \text { if } 0<\alpha \leq 1, \\
\int_{0}^{\infty} K_{\alpha}(r) d r=1-2 / \alpha \text { if } 1<\alpha \leq 2 .
\end{gathered}
$$

In Figs. 3 and 4 we display the plots of $K_{\alpha}(r)$, that we denote as the basic spectral function, for some values of $\alpha$ in the intervals (a) $0<\alpha<1$, (b) $1<\alpha<2$.

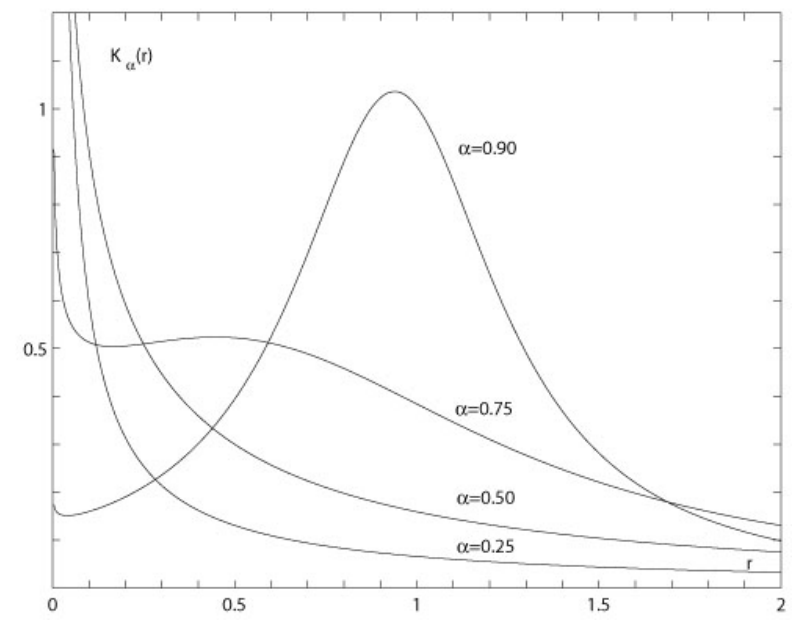

Figure 3: Plots of the basic spectral function $K_{\alpha}(r)$ for $0<\alpha<1: \alpha=0.25,0.50,0.75,0.90$.

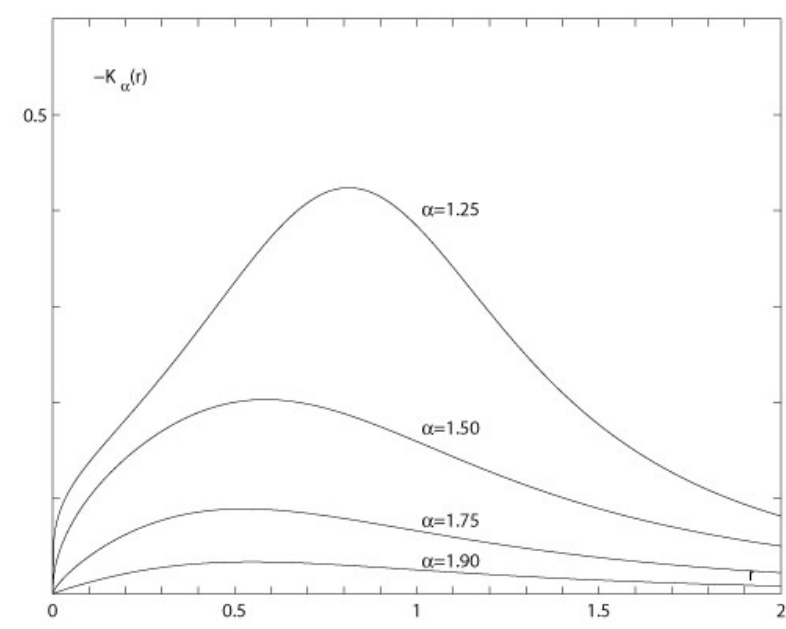

Figure 4: Plots of the basic spectral function $-K_{\alpha}(r)$ for $1<\alpha<2: \alpha=1.25,1.50,1.75, .1 .90$.

In addition to the basic fundamental solutions, $u_{0}(t)=e_{\alpha}(t)$, we need to compute the impulseresponse solutions $u_{\delta}(t)=-D^{1} e_{\alpha}(t)$ for cases (a) and (b) and, only in case (b), the second fundamental solution $u_{1}(t)=J^{1} e_{\alpha}(t)$. 
For this purpose we note that in general it turns out that

$$
J^{k} f_{\alpha}(t)=\int_{0}^{\infty} \mathrm{e}^{-r t} K_{\alpha}^{k}(r) d r
$$

with

$$
\begin{aligned}
K_{\alpha}^{k}(r) & :=(-1)^{k} r^{-k} K_{\alpha}(r) \\
& =\frac{(-1)^{k}}{\pi} \frac{r^{\alpha-1-k} \sin (\alpha \pi)}{r^{2 \alpha}+2 r^{\alpha} \cos (\alpha \pi)+1},
\end{aligned}
$$

where $K_{\alpha}(r)=K_{\alpha}^{0}(r)$, and

$$
J^{k} g_{\alpha}(t)=\frac{2}{\alpha} \mathrm{e}^{t \cos (\pi / \alpha)} \cos \left[t \sin \left(\frac{\pi}{\alpha}\right)-k \frac{\pi}{\alpha}\right] .
$$

For the impulse-response solution we note that the effect of the differential operator $D^{1}$ is the same as that of the virtual operator $J^{-1}$.

Hence the solutions for the fractional relaxation are: (a) $0<\alpha<1$,

$$
u(t)=c_{0} u_{0}(t)+\int_{0}^{t} q(t-\tau) u_{\delta}(\tau) d \tau,
$$

where

$$
\begin{aligned}
& u_{0}(t)=\int_{0}^{\infty} \mathrm{e}^{-r t} K_{\alpha}^{0}(r) d r, \\
& u_{\delta}(t)=-\int_{0}^{\infty} \mathrm{e}^{-r t} K_{\alpha}^{-1}(r) d r,
\end{aligned}
$$

with

$$
u_{0}\left(0^{+}\right)=1, u_{\delta}\left(0^{+}\right)=\infty,
$$

and for $t \rightarrow \infty$

$$
u_{0}(t) \sim \frac{t^{-\alpha}}{\Gamma(1-\alpha)}, u_{1}(t) \sim \frac{t^{1-\alpha}}{\Gamma(2-\alpha)} .
$$

Hence the solutions for the fractional oscillation are: (b) $1<\alpha<2$,

$$
\begin{aligned}
u(t)=c_{0} & u_{0}(t)+c_{1} u_{1}(t)+\int_{0}^{t} q(t-\tau) u_{\delta}(\tau) d \tau, \\
u_{0}(t) & =\int_{0}^{\infty} \mathrm{e}^{-r t} K_{\alpha}^{0}(r) d r \\
& +\frac{2}{\alpha} \mathrm{e}^{t \cos (\pi / \alpha)} \cos \left[t \sin \left(\frac{\pi}{\alpha}\right)\right], \\
u_{1}(t) & =\int_{0}^{\infty} \mathrm{e}^{-r t} K_{\alpha}^{1}(r) d r \\
& +\frac{2}{\alpha} \mathrm{e}^{t \cos (\pi / \alpha)} \cos \left[t \sin \left(\frac{\pi}{\alpha}\right)-\frac{\pi}{\alpha}\right], \\
& =-\int_{0}^{\infty} \mathrm{e}^{-r t} K_{\alpha}^{-1}(r) d r \\
& -\frac{2}{\alpha} \mathrm{e}^{t \cos (\pi / \alpha)} \cos \left[t \sin \left(\frac{\pi}{\alpha}\right)+\frac{\pi}{\alpha}\right],
\end{aligned}
$$

with

$$
\begin{gathered}
u_{0}\left(0^{+}\right)=1, u_{0}^{\prime}\left(0^{+}\right)=0, \\
u_{1}\left(0^{+}\right)=0, u_{1}^{\prime}\left(0^{+}\right)=1, \\
u_{\delta}\left(0^{+}\right)=0, u_{\delta}^{\prime}\left(0^{+}\right)=+\infty,
\end{gathered}
$$

and for $t \rightarrow \infty$

$$
\left\{\begin{aligned}
u_{0}(t) & \sim \frac{t^{-\alpha}}{\Gamma(1-\alpha)}, \\
u_{1}(t) & \sim \frac{t^{1-\alpha}}{\Gamma(2-\alpha)}, \\
u_{\delta}(t) & \sim-\frac{t^{-\alpha-1}}{\Gamma(-\alpha)}
\end{aligned}\right.
$$

In Figs. 2a and $2 \mathrm{~b}$ we display the plots of the basic fundamental solution for the following cases, respectively :

(a) $\alpha=0.25,0.50,0.75,1$,

(b) $\alpha=1.25,1.50,1.75,2$,

obtained from the first formula in (5.13a) and (5.13b), respectively.

We now want to point out that in both the cases (a) and (b) (in which $\alpha$ is just not integer) i.e. for fractional relaxation and fractional oscillation, all the fundamental and impulse-response solutions exhibit an algebraic decay as $t \rightarrow \infty$, as discussed above.

This algebraic decay is the most important effect of the non-integer derivative in our equations, which dramatically differs from the exponential decay present in the ordinary relaxation and damped-oscillation phenomena.

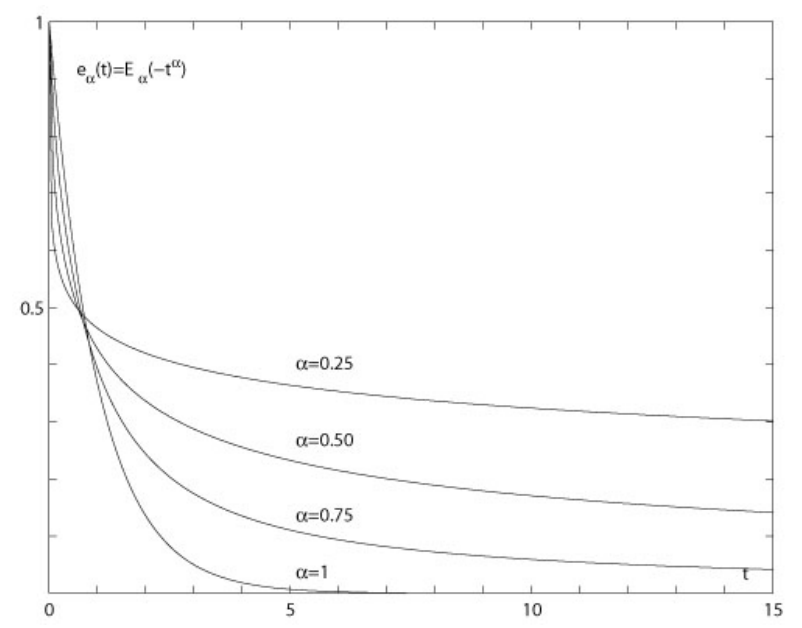

Figure 5: Plots of the basic fundamental solution $u_{0}(t)=e_{\alpha}(t)$ with $\alpha=0.25,0.50,0.75,1$. 


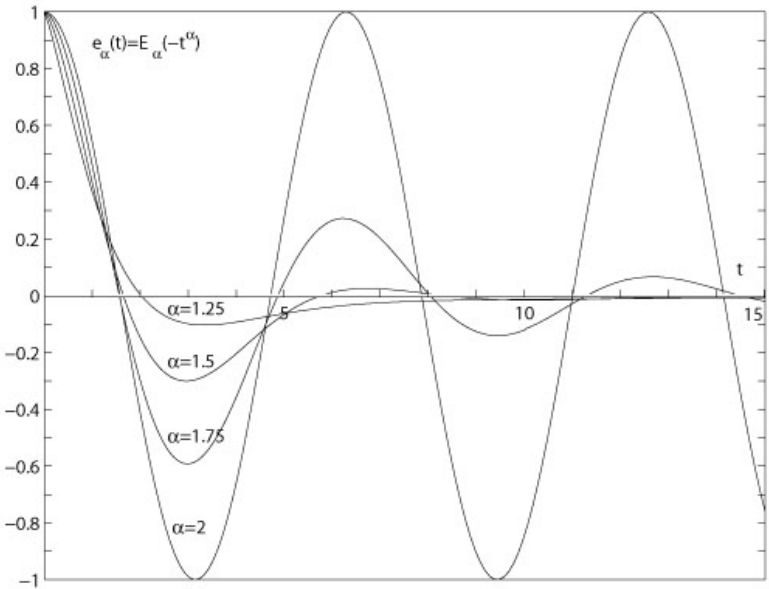

Figure 6: Plots of the basic fundamental solution $u_{0}(t)=e_{\alpha}(t)$ with $\alpha=1.25,1.50,1.75,2$.

We would like to remark the difference between fractional relaxation governed by the Mittag-Leffler type function $E_{\alpha}\left(-a t^{\alpha}\right)$ and stretched relaxation governed by a stretched exponential function $\exp \left(-b t^{\alpha}\right)$ with $\alpha, a, b>0$ for $t \geq 0$. A common behaviour is achieved only in a restricted range $0 \leq t \ll 1$ where

$$
\begin{aligned}
E_{\alpha}\left(-a t^{\alpha}\right) & \simeq 1-\frac{a}{\Gamma(\alpha+1)} t^{\alpha}=1-b t^{\alpha} \\
& \simeq \mathrm{e}^{-b t^{\alpha}}, b=\frac{a}{\Gamma(\alpha+1)} .
\end{aligned}
$$

In Figs. 3a, 3b, 3c for $\alpha=0.25,0.50,0.75$ we have compared $E_{\alpha}\left(-t^{\alpha}\right)$ (full line) with its asymptotic approximations $\exp \left[-t^{\alpha} / \Gamma(1+\alpha)\right]$ (dashed line) valid for short times, and $t^{-\alpha} / \Gamma(1-\alpha)$ (dotted line) valid for long times.

We have adopted log-log plots in order to better achieve such a comparison and the transition from the stretched exponential to the inverse power-law decay.

In Figs. 4a, 4b, 4c we have shown some plots of the basic fundamental solution $u_{0}(t)=e_{\alpha}(t)$ for $\alpha=$ $1.25,1.50,1.75$, respectively.

Here the algebraic decay of the fractional oscillation can be recognized and compared with the two contributions provided by $f_{\alpha}$ (monotonic behaviour, dotted line) and $g_{\alpha}(t)$ (exponentially damped oscillation, dashed line)

\section{The zeros of the solutions of the fractional oscillation}

Now we find it interesting to carry out some investigations about the zeros of the basic fundamental so-
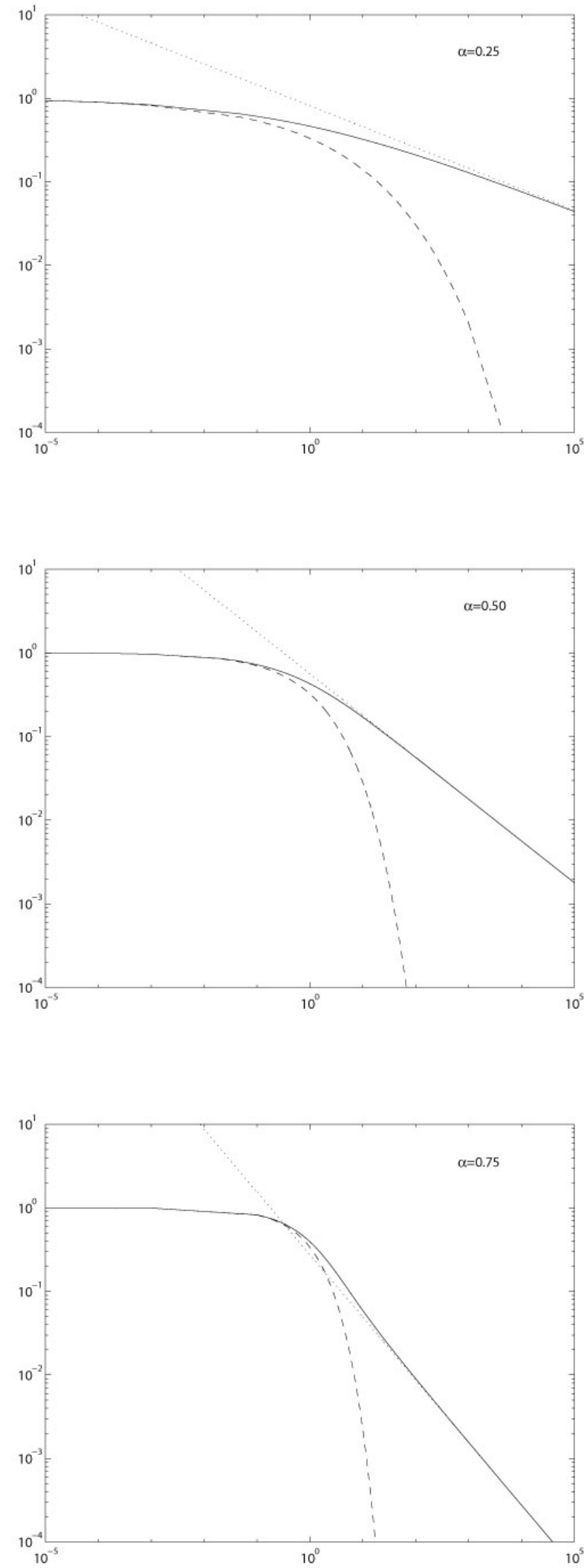

Figure 7: $\log -\log$ plot of $E_{\alpha}\left(-t^{\alpha}\right)$ for $\alpha=$ $0.25,0.50,0.75$. 

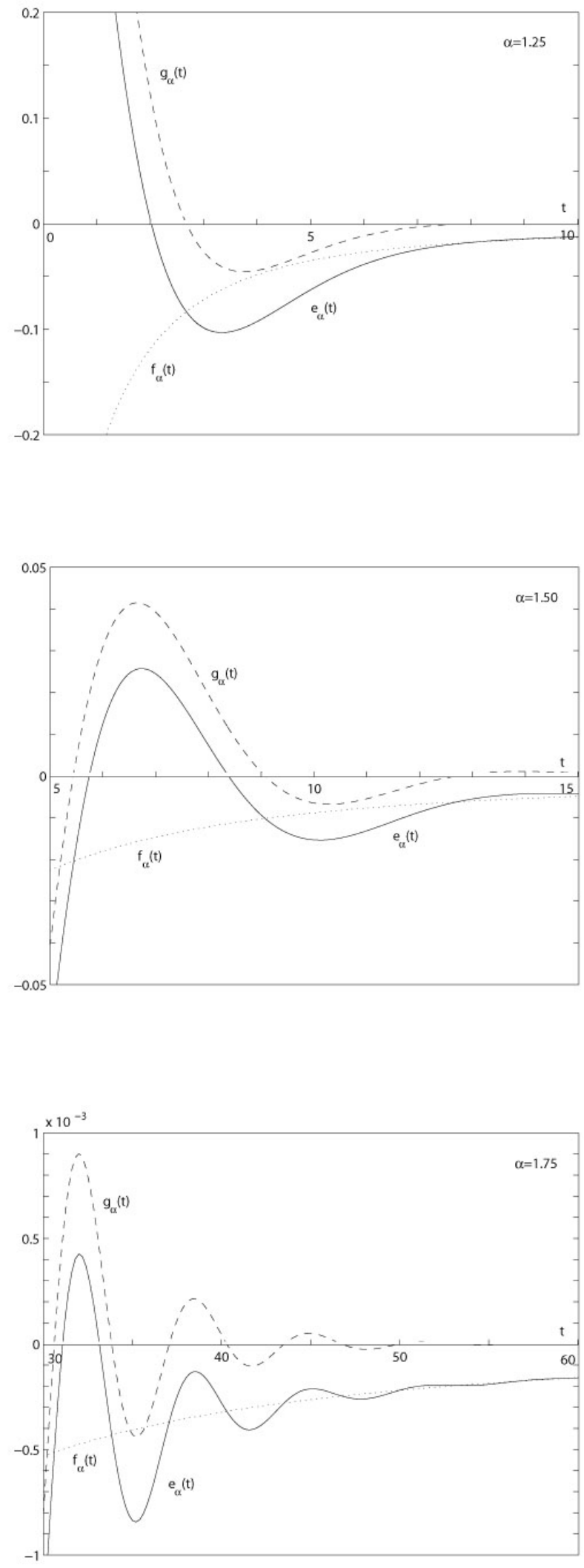

Figure 8: Decay of the basic fundamental solution $u_{0}(t)=e_{\alpha}(t)$ for $\alpha=1.25,1.50,1.75$; full line $=$ $e_{\alpha}(t)$, dashed line $=g_{\alpha}(t)$, dotted line $=f_{\alpha}(t)$. lution $u_{0}(t)=e_{\alpha}(t)$ in the case (b) of fractional oscillations. For the second fundamental solution and the impulse-response solution the analysis of the zeros can be easily carried out analogously.

Recalling the first equation in (5.13b), the required zeros of $e_{\alpha}(t)$ are the solutions of the equation

$e_{\alpha}(t)=f_{\alpha}(t)+\frac{2}{\alpha} \mathrm{e}^{t \cos (\pi / \alpha)} \cos \left[t \sin \left(\frac{\pi}{\alpha}\right)\right]=0$.

We first note that the function $e_{\alpha}(t)$ exhibits an odd number of zeros, in that $e_{\alpha}(0)=1$, and, for sufficiently large $t, e_{\alpha}(t)$ turns out to be permanently negative, as shown in (5.14b) by the sign of $\Gamma(1-\alpha)$.

The smallest zero lies in the first positivity interval of $\cos [t \sin (\pi / \alpha)]$, hence in the interval $0<t<$ $\pi /[2 \sin (\pi / \alpha)] ;$ all other zeros can only lie in the succeeding positivity intervals of $\cos [t \sin (\pi / \alpha)]$, in each of these two zeros are present as long as

$$
\frac{2}{\alpha} \mathrm{e}^{t \cos (\pi / \alpha)} \geq\left|f_{\alpha}(t)\right| .
$$

When $t$ is sufficiently large the zeros are expected to be found approximately from the equation

$$
\frac{2}{\alpha} \mathrm{e}^{t \cos (\pi / \alpha)} \approx \frac{t^{-\alpha}}{|\Gamma(1-\alpha)|},
$$

obtained from (5.15) by ignoring the oscillation factor of $g_{\alpha}(t)$ and taking the first term in the asymptotic expansion of $f_{\alpha}(t)$. As shown in the report [18] such approximate equation turns out to be useful when $\alpha \rightarrow 1^{+}$and $\alpha \rightarrow 2^{-}$.

For $\alpha \rightarrow 1^{+}$, only one zero is present, which is expected to be very far from the origin in view of the large period of the function $\cos [t \sin (\pi / \alpha)]$. In fact, since there is no zero for $\alpha=1$, and by increasing $\alpha$ more and more zeros arise, we are sure that only one zero exists for $\alpha$ sufficiently close to 1 . Putting $\alpha=1+\epsilon$ the asymptotic position $T_{*}$ of this zero can be found from the relation (5.17) in the limit $\epsilon \rightarrow 0^{+}$. Assuming in this limit the first-order approximation, we get

$$
T_{*} \sim \log \left(\frac{2}{\epsilon}\right),
$$

which shows that $T_{*}$ tends to infinity slower than $1 / \epsilon$, as $\epsilon \rightarrow 0$. For details see again the 1995 report by Gorenflo \& Mainardi [18].

For $\alpha \rightarrow 2^{-}$, there is an increasing number of zeros up to infinity since $e_{2}(t)=\cos t$ has infinitely many zeros [in $\left.t_{n}^{*}=(n+1 / 2) \pi, n=0,1, \ldots\right]$. Putting now $\alpha=2-\delta$ the asymptotic position $T_{*}$ for the 
largest zero can be found again from (5.17) in the limit $\delta \rightarrow 0^{+}$. Assuming in this limit the first-order approximation, we get

$$
T_{*} \sim \frac{12}{\pi \delta} \log \left(\frac{1}{\delta}\right) .
$$

Now, for $\delta \rightarrow 0^{+}$the length of the positivity intervals of $g_{\alpha}(t)$ tends to $\pi$ and, as long as $t \leq T_{*}$, there are two zeros in each positivity interval. Hence, in the limit $\delta \rightarrow 0^{+}$, there is in average one zero per interval of length $\pi$, so we expect that $N_{*} \sim T_{*} / \pi$.

Remark : For the above considerations we got inspiration from an interesting paper by Wiman (1905) [60], who at the beginning of the XX-th century, after having treated the Mittag-Leffler function in the complex plane, considered the position of the zeros of the function on the negative real axis (without providing any detail). The expressions of $T_{*}$ are in disagreement with those by Wiman for numerical factors; however, the results of our numerical studies carried out in the 1995 report [18] confirm and illustrate the validity of the present analysis.

Here, we find it interesting to analyse the phenomenon of the transition of the (odd) number of zeros as $1.4 \leq$ $\alpha \leq 1.8$. For this purpose, in Table I we report the intervals of amplitude $\Delta \alpha=0.01$ where these transitions occur, and the location $T_{*}$ (evaluated within a relative error of $0.1 \%$ ) of the largest zeros found at the two extreme values of the above intervals.

We recognize that the transition from 1 to 3 zeros occurs as $1.40 \leq \alpha \leq 1.41$, that one from 3 to $5 \mathrm{ze}$ ros occurs as $1.56 \leq \alpha \leq 1.57$, and so on. The last transition is from 15 to 17 zeros, and it just occurs as $1.79 \leq \alpha \leq 1.80$.

\begin{tabular}{|c|c|c|}
\hline$N_{*}$ & $\alpha$ & $T_{*}$ \\
\hline $1 \div 3$ & $1.40 \div 1.41$ & $1.730 \div 5.726$ \\
\hline $3 \div 5$ & $1.56 \div 1.57$ & $8.366 \div 13.48$ \\
\hline $5 \div 7$ & $1.64 \div 1.65$ & $14.61 \div 20.00$ \\
\hline $7 \div 9$ & $1.69 \div 1.70$ & $20.80 \div 26.33$ \\
\hline $9 \div 11$ & $1.72 \div 1.73$ & $27.03 \div 32.83$ \\
\hline $11 \div 13$ & $1.75 \div 1.76$ & $33.11 \div 38.81$ \\
\hline $13 \div 15$ & $1.78 \div 1.79$ & $39.49 \div 45.51$ \\
\hline $15 \div 17$ & $1.79 \div 1.80$ & $45.51 \div 51.46$ \\
\hline
\end{tabular}

Table I

$$
\begin{gathered}
N_{*}=\text { number of zeros, } \alpha=\text { fractional order } \\
T_{*} \text { location of the largest zero. }
\end{gathered}
$$

\section{The functions of the Wright type}

The classical Wright function, that we denote by $W_{\lambda, \mu}(z)$, is defined by the series representation convergent on the whole complex plane $\mathbb{C}$,

$W_{\lambda, \mu}(z)=\sum_{n=0}^{\infty} \frac{z^{n}}{n ! \Gamma(\lambda n+\mu)}, \lambda>-1, \mu \in \mathbb{C}$.

One of its integral representations for $\lambda>-1, \mu \in \mathbb{C}$ reads as:

$$
W_{\lambda, \mu}(z)=\frac{1}{2 \pi i} \int_{H a} \mathrm{e}^{\sigma+z \sigma^{-\lambda}} \frac{d \sigma}{\sigma^{\mu}},
$$

where, as usual, $H a$ denotes the Hankel path. Then, $W_{\lambda, \mu}(z)$ is an entire function for all $\lambda \in(-1,+\infty)$. Originally, in 1930's Wright assumed $\lambda \geq 0$ in connection with his investigations on the asymptotic theory of partitions [63, 64], and only in 1940 [65] he considered $-1<\lambda<0$.

We note that in the Vol 3, Chapter 18 of the handbook of the Bateman Project [10], presumably for a misprint, the parameter $\lambda$ is restricted to be non-negative, whereas the Wright functions remained practically ignored in other handbooks. In 1990's Mainardi, being aware only of the Bateman handbook, proved that the Wright function is entire also for $-1<\lambda<0$ in his approaches to the time fractional diffusion equation, see $[28,29,30]$.

In view of the asymptotic representation in the complex domain and of the Laplace transform for positive argument $z=r>0$ ( $r$ can denote the time variable $t$ or the positive space variable $x$ ) the Wright functions are distinguished in first kind $(\lambda \geq 0)$ and second kind $(-1<\lambda<0)$ as outlined in the Appendix $\mathrm{F}$ of the book by Mainardi [32].

It is possible to prove that the Wright function is entire of order $1 /(1+\lambda)$, hence of exponential type if $\lambda \geq 0$., that is only for the Wright functions of the first kind. The case $\lambda=0$ is trivial since $W_{0, \mu}(z)=\mathrm{e}^{z} / \Gamma(\mu)$.

\section{Recurrence relations}

Some of the properties, that the Wright functions share with the most popular Bessel functions, were enumerated by Wright himself.

Hereafter, we quote some relevant relations from the handbook of Bateman Project Handbook [10]:

$$
\begin{gathered}
\lambda z W_{\lambda, \lambda+\mu}(z)=W_{\lambda, \mu-1}(z)+(1-\mu) W_{\lambda, \mu}(z), \\
\frac{d}{d z} W_{\lambda, \mu}(z)=W_{\lambda, \lambda+\mu}(z) .
\end{gathered}
$$


We note that these relations can easily be derived from the series or integral representations, (6.1) or (6.2).

\section{Generalization of the Bessel functions.}

For $\lambda=1$ and $\mu=\nu+1 \geq 0$ the Wright functions (of the first kind) turn out to be related to the well known Bessel functions $J_{\nu}$ and $I_{\nu}$ by the identities:

$$
\begin{aligned}
& J_{\nu}(z)=\left(\frac{z}{2}\right)^{\nu} W_{1, \nu+1}\left(-\frac{z^{2}}{4}\right), \\
& I_{\nu}(z)=\left(\frac{z}{2}\right)^{\nu} W_{1, \nu+1}\left(\frac{z^{2}}{4}\right) .
\end{aligned}
$$

In view of this property some authors refer to the Wright function as the Wright generalized Bessel function (misnamed also as the Bessel-Maitland function) and introduce the notation

$$
\begin{aligned}
J_{\nu}^{(\lambda)}(z) & :=\left(\frac{z}{2}\right)^{\nu} W_{\lambda, \nu+1}\left(-\frac{z^{2}}{4}\right) \\
& =\left(\frac{z}{2}\right)^{\nu} \sum_{n=0}^{\infty} \frac{(-1)^{n}(z / 2)^{2 n}}{n ! \Gamma(\lambda n+\nu+1)},
\end{aligned}
$$

with $\lambda>0$ and $\nu>-1$. In particular $J_{\nu}^{(1)}(z):=$ $J_{\nu}(z)$. As a matter of fact, the Wright functions (of the first kind) appear as the natural generalization of the entire functions known as Bessel - Clifford functions, see e.g. Kiryakova [23], and referred by Tricomi [58] as the uniform Bessel functions, see also Gatteschi [13].

Similarly we can properly define $I_{\nu}^{(\lambda)}(z)$.

\subsection{The Mainardi auxiliary functions}

We note that two particular Wright functions of the second kind, were introduced by Mainardi in 1990's $[28,29,30]$ named $F_{\nu}(z)$ and $M_{\nu}(z)(0<\nu<1)$, called auxiliary functions in virtue of their role in the time fractional diffusion equations. These functions are indeed special cases of the Wright function of the second kind $W_{\lambda, \mu}(z)$ by setting, respectively, $\lambda=-\nu$ and $\mu=0$ or $\mu=1-\nu$. Hence we have:

$$
F_{\nu}(z):=W_{-\nu, 0}(-z), \quad 0<\nu<1,
$$

and

$$
M_{\nu}(z):=W_{-\nu, 1-\nu}(-z), \quad 0<\nu<1,
$$

These functions are interrelated through the following relation:

$$
F_{\nu}(z)=\nu z M_{\nu}(z)
$$

The series and integral representations of the auxiliary functions are derived from those of the general Wright functions. Then for $z \in \mathbb{C}$ and $0<\nu<1$ we have:

$$
\begin{aligned}
F_{\nu}(z) & =\sum_{n=1}^{\infty} \frac{(-z)^{n}}{n ! \Gamma(-\nu n)} \\
& =\frac{1}{\pi} \sum_{n=1}^{\infty} \frac{(-z)^{n-1}}{n !} \Gamma(\nu n+1) \sin (\pi \nu n),
\end{aligned}
$$

and

$$
\begin{aligned}
M_{\nu}(z) & =\sum_{n=0}^{\infty} \frac{(-z)^{n}}{n ! \Gamma[-\nu n+(1-\nu)]} \\
& =\frac{1}{\pi} \sum_{n=1}^{\infty} \frac{(-z)^{n-1}}{(n-1) !} \Gamma(\nu n) \sin (\pi \nu n),
\end{aligned}
$$

The second series representations in (6.10)-(6.11) have been obtained by using the well-known reflection formula for the Gamma function,

$$
\Gamma(\zeta) \Gamma(1-\zeta)=\pi / \sin \pi \zeta .
$$

For the integral representation we have

$$
F_{\nu}(z):=\frac{1}{2 \pi i} \int_{H a} \mathrm{e}^{\sigma-z \sigma^{\nu}} d \sigma
$$

and

$$
M_{\nu}(z):=\frac{1}{2 \pi i} \int_{H a} \mathrm{e}^{\sigma-z \sigma^{\nu}} \frac{d \sigma}{\sigma^{1-\nu}} .
$$

As usual, the equivalence of the series and integral representations is easily proved using the Hankel formula for the Gamma function and performing a termby-term integration.

Explicit expressions of $F_{\nu}(z)$ and $M_{\nu}(z)$ in terms of known functions are expected for some particular values of $\nu$ as shown and recalled in [28, 29, 30], that is

$$
\begin{array}{r}
M_{1 / 2}(z)=\frac{1}{\sqrt{\pi}} \mathrm{e}^{-z^{2} / 4}, \\
M_{1 / 3}(z)=3^{2 / 3} \operatorname{Ai}\left(z / 3^{1 / 3}\right), .
\end{array}
$$

Liemert and Klenie [24] have added the following expression for $\nu=2 / 3$

$$
\begin{aligned}
M_{2 / 3}(z) & =3^{-2 / 3} \mathrm{e}^{-2 z^{3} / 27} \\
& {\left[3^{1 / 3} z \mathrm{Ai}\left(z^{2} / 3^{4 / 3}\right)-3 \mathrm{Ai}^{\prime}\left(z^{2} / 3^{4 / 3}\right)\right] }
\end{aligned}
$$

where $\mathrm{Ai}$ and $\mathrm{Ai}^{\prime}$ denote the Airy function and its first derivative. Furthermore they have suggested in the positive real field $\mathbb{R}^{+}$the following remarkably integral representation

$$
\begin{aligned}
M_{\nu}(x) & =\frac{1}{\pi} \frac{x^{\nu /(1-\nu)}}{1-\nu} \\
& \cdot \int_{0}^{\pi} C_{\nu}(\phi) \exp \left(-C_{\nu}(\phi)\right) x^{1 /(1-\nu)} d \phi,
\end{aligned}
$$


where

$$
C_{\nu}(\phi)=\frac{\sin (1-\nu)}{\sin \phi}\left(\frac{\sin \nu \phi}{\sin \phi}\right)^{\nu /(1-\nu)}
$$

corresponding to equation (7) of the article written by Saa and Venegeroles [53] .

Furthermore, it can be proved, see [41] that $M_{1 / q}(z)$ satisfies the differential equation of order $q-1$

$$
\frac{d^{q-1}}{d z^{q-1}} M_{1 / q}(z)+\frac{(-1)^{q}}{q} z M_{1 / q}(z)=0,
$$

subjected to the $q-1$ initial conditions at $z=0$, derived from (6.15),

$$
M_{1 / q}^{(h)}(0)=\frac{(-1)^{h}}{\pi} \Gamma[(h+1) / q] \sin [\pi(h+1) / q],
$$

with $h=0,1, \ldots q-2$. We note that, for $q \geq 4$, Eq. (6.18) is akin to the hyper-Airy differential equation of order $q-1$, see e.g. [Bender \& Orszag 1987].

We find it convenient to show the plots of the $M$ Wright functions on a space symmetric interval of $\mathbb{R}$ in Figs 1, 2, corresponding to the cases $0 \leq \nu \leq 1 / 2$ and $1 / 2 \leq \nu \leq 1$, respectively. We recognize the non-negativity of the $M$-Wright function on $\mathbb{R}$ for $1 / 2 \leq \nu \leq 1$ consistently with the analysis on distribution of zeros and asymptotics of Wright functions carried out by Luchko, see [25], [26].

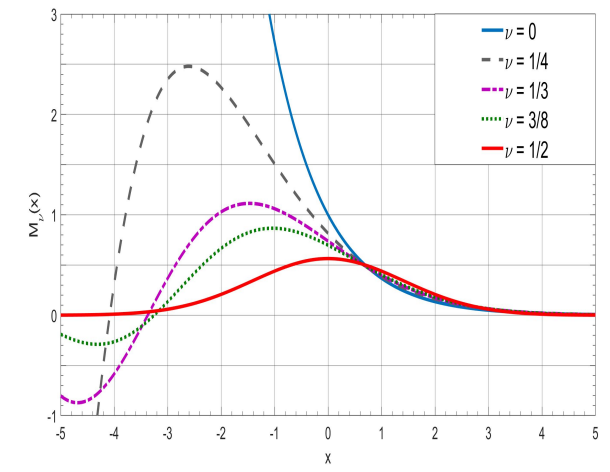

Figure 9: Plots of the $M$-Wright function as a function of the $x$ variable, for $0 \leq \nu \leq 1 / 2$.

\subsection{Laplace transform pairs related to the Wright function}

Let us consider the Laplace transform of the Wright function using the usual notation

$$
W_{\lambda, \mu}( \pm r) \div \int_{0}^{\infty} \mathrm{e}^{-s r} W_{\lambda, \mu}( \pm r) d r
$$

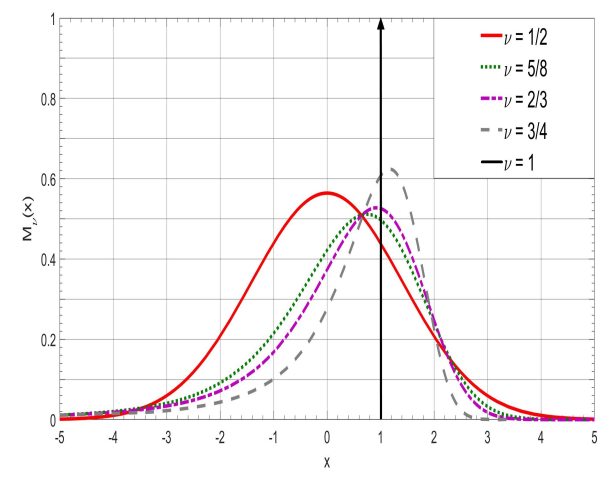

Figure 10: Plots of the $M$-Wright function as a function of the $x$ variable, for $1 / 2 \leq \nu \leq 1$.

where $r$ denotes a non negative real variable and $s$ is the Laplace complex parameter.

When $\lambda>0$ the series representation of the Wright function can be transformed term-by-term. In fact, for a known theorem of the theory of the Laplace transforms, see e.g. Doetsch (194) [8], the Laplace transform of an entire function of exponential type can be obtained by transforming term-by-term the Taylor expansion of the original function around the origin. In this case the resulting Laplace transform turns out to be analytic and vanishing at infinity. As a consequence, we obtain the Laplace transform pair for $|s|>0$

$$
W_{\lambda, \mu}( \pm r) \div \frac{1}{s} E_{\lambda, \mu}\left( \pm \frac{1}{s}\right), \lambda>0,
$$

where $E_{\lambda, \mu}$ denotes the Mittag-Leffler function in two parameters. The proof is straightforward noting that

$$
\sum_{n=0}^{\infty} \frac{( \pm r)^{n}}{n ! \Gamma(\lambda n+\mu)} \div \frac{1}{s} \sum_{n=0}^{\infty} \frac{( \pm 1 / s)^{n}}{\Gamma(\lambda n+\mu)},
$$

and recalling the series representation of the MittagLeffler function,

$$
E_{\alpha, \beta}(z):=\sum_{n=0}^{\infty} \frac{z^{n}}{\Gamma(\alpha n+\beta)}, \alpha>0, \beta \in \mathbb{C} .
$$

For $\lambda \rightarrow 0^{+}$Eq. (6.20) provides the Laplace transform pair for $|s|>0$,

$$
\begin{aligned}
& W_{0^{+}, \mu}( \pm r)=\frac{\mathrm{e}^{ \pm r}}{\Gamma(\mu)} \\
& \div \frac{1}{\Gamma(\mu)} \frac{1}{s \mp 1}=\frac{1}{s} E_{0, \mu}\left( \pm \frac{1}{s}\right)
\end{aligned},
$$

where, to remain in agreement with (6.20), we have formally put,

$E_{0, \mu}(z):=\sum_{n=0}^{\infty} \frac{z^{n}}{\Gamma(\mu)}:=\frac{1}{\Gamma(\mu)} E_{0}(z):=\frac{1}{\Gamma(\mu)} \frac{1}{1-z}$. 
We recognize that in this special case the Laplace transform exhibits a simple pole at $s= \pm 1$ while for $\lambda>0$ it exhibits an essential singularity at $s=0$.

For $-1<\lambda<0$ the Wright function turns out to be an entire function of order greater than 1 , so that care is required in establishing the existence of its Laplace transform, which necessarily must tend to zero as $s \rightarrow$ $\infty$ in its half-plane of convergence.

For the sake of convenience we limit ourselves to derive the Laplace transform for the special case of $M_{\nu}(r)$; the exponential decay as $r \rightarrow \infty$ of the original function provided by (6.20) ensures the existence of the image function. From the integral representation (6.13) of the $M_{\nu}$ function we obtain

$$
\begin{gathered}
M_{\nu}(r) \div \frac{1}{2 \pi i} \int_{0}^{\infty} \mathrm{e}^{-s r}\left[\int_{H a} \mathrm{e}^{\sigma-r \sigma^{\nu}} \frac{d \sigma}{\sigma^{1-\nu}}\right] d r \\
=\frac{1}{2 \pi i} \int_{H a} \mathrm{e}^{\sigma} \sigma^{\nu-1}\left[\int_{0}^{\infty} \mathrm{e}^{-r\left(s+\sigma^{\nu}\right)} d r\right] d \sigma \\
=\frac{1}{2 \pi i} \int_{H a} \frac{\mathrm{e}^{\sigma} \sigma^{\nu-1}}{\sigma^{\nu}+s} d \sigma .
\end{gathered}
$$

Then, by recalling the integral representation of the Mittag-Leffler function (3.4),

$$
E_{\alpha}(z)=\frac{1}{2 \pi i} \int_{H a} \frac{\zeta^{\alpha-1} \mathrm{e}^{\zeta}}{\zeta^{\alpha}-z} d \zeta, \alpha>0, z \in \mathbb{C},
$$

we obtain the Laplace transform pair

$$
\begin{aligned}
& M_{\nu}(r):=W_{-\nu, 1-\nu}(-r) \\
& \div E_{\nu}(-s), 0<\nu<1, .
\end{aligned}
$$

In this case, transforming term-by-term the Taylor series of $M_{\nu}(r)$ yields a series of negative powers of $s$, that represents the asymptotic expansion of $E_{\nu}(-s)$ as $s \rightarrow \infty$ in a sector around the positive real axis.

We note that (6.22) contains the well-known Laplace transform pair, see e.g. Doetsch [8] and Eq. (3.7):

$$
\begin{aligned}
M_{1 / 2}(r) & :=\frac{1}{\sqrt{\pi}} \exp \left(-r^{2} / 4\right) \\
& \div E_{1 / 2}(-s)=\exp \left(s^{2}\right) \operatorname{erfc}(s),
\end{aligned}
$$

valid $\forall s \in \mathbb{C}$

Analogously, using the more general integral representation (6.2) of the standard Wright function, we can prove that in the case $\lambda=-\nu \in(-1,0)$ and $\operatorname{Re}(\mu)>0$, we get

$$
W_{-\nu, \mu}(-r) \div E_{\nu, \mu+\nu}(-s), 0<\nu<1 .
$$

In the limit as $\nu \rightarrow 0^{+}$(thus $\lambda \rightarrow 0^{-}$) we formally obtain the Laplace transform pair

$$
\begin{aligned}
W_{0^{-}, \mu}(-r) & :=\frac{\mathrm{e}^{-r}}{\Gamma(\mu)} \\
& \div \frac{1}{\Gamma(\mu)} \frac{1}{s+1}:=E_{0, \mu}(-s)
\end{aligned}
$$

Therefore, as $\lambda \rightarrow 0^{ \pm}$, and $\mu=1$ we note a sort of continuity in the results (6.21) and (6.25) since

$$
W_{0,1}(-r):=\mathrm{e}^{-r} \div \frac{1}{(s+1)}
$$

with

$$
\frac{1}{(s+1)}=\left\{\begin{array}{l}
(1 / s) E_{0}(-1 / s),|s|>1 ; \\
E_{0}(-s),|s|<1 .
\end{array}\right.
$$

We here point out the relevant Laplace transform pairs related to the auxiliary functions of argument $r^{-\nu}$ with $0<\nu<1$, see for details the cited author's papers

$$
\begin{aligned}
& \frac{1}{r} F_{\nu}\left(1 / r^{\nu}\right)=\frac{\nu}{r^{\nu+1}} M_{\nu}\left(1 / r^{\nu}\right) \div \mathrm{e}^{-s^{\nu}}, \\
& \frac{1}{\nu} F_{\nu}\left(1 / r^{\nu}\right)=\frac{1}{r^{\nu}} M_{\nu}\left(1 / r^{\nu}\right) \div \frac{\mathrm{e}^{-s^{\nu}}}{s^{1-\nu}} .
\end{aligned}
$$

We recall that the Laplace transform pairs in (6.28) were formerly considered by Pollard (1946) [50], Later Mikusinski (1959 )[43] got a similar result based on his theory of operational calculus, and finally, albeit unaware of the previous results, Buchen \& Mainardi (1975) [2] derived the result in a formal way. We note, however, that all these Authors were not informed about the Wright functions. Aware of the Wright functions was Stankovic [57] who in 1970 gave a rigorous proof of the Laplace transform pairs involving the Wright functions with first negative parameter, here referred of the second kind,

Hereafter we like to provide two independent proofs of (6.28) carrying out the inversion of $\exp \left(-s^{\nu}\right)$, either by the complex Bromwich integral formula or by the formal series method. Similarly we can act for the Laplace transform pair (6.29).

For the complex integral approach we deform the Bromwich path $\mathrm{Br}$ into the Hankel path $\mathrm{Ha}$, that is equivalent to the original path, and we set $\sigma=s r$. Recalling (6.13)-(6.14), we get

$$
\mathcal{L}^{-1}\left[\exp \left(-s^{\nu}\right)\right]=\frac{1}{2 \pi i} \int_{B r} \mathrm{e}^{s r-s^{\nu}} d s
$$




$$
\begin{gathered}
=\frac{1}{2 \pi i r} \int_{H a} \mathrm{e}^{\sigma-(\sigma / r)^{\nu}} d \sigma \\
=\frac{1}{r} F_{\nu}\left(1 / r^{\nu}\right)=\frac{\nu}{r^{\nu+1}} M_{\nu}\left(1 / r^{\nu}\right) .
\end{gathered}
$$

Expanding in power series the Laplace transform and inverting term by term we formally get, after recalling (6.12)-(6.13):

$$
\begin{gathered}
\mathcal{L}^{-1}\left[\exp \left(-s^{\nu}\right)\right]=\sum_{n=0}^{\infty} \frac{(-1)^{n}}{n !} \mathcal{L}^{-1}\left[s^{\nu n}\right] \\
=\sum_{n=1}^{\infty} \frac{(-1)^{n}}{n !} \frac{r^{-\nu n-1}}{\Gamma(-\nu n)} \\
=\frac{1}{r} F_{\nu}\left(1 / r^{\nu}\right)=\frac{\nu}{r^{\nu+1}} M_{\nu}\left(1 / r^{\nu}\right) .
\end{gathered}
$$

We note the relevance of Laplace transforms (6.24) and (6.28) in pointing out the non-negativity of the Wright function $M_{\nu}(x)$ for $x>0$ and the complete monotonicity of the Mittag-leffler functions $E_{\nu}(-x)$ for $x>0$ and $0<\nu<1$. In fact, since $\exp \left(-s^{\nu}\right)$ denotes the Laplace transform of a probability density (precisely, the extremal Lévy stable density of index $\nu$, see [Feller (1971)]), the L.H.S. of (6.28) must be non-negative, and so also the L.H.S of $\mathrm{F}(24)$. As a matter of fact the Laplace transform pair (6.24) shows, replacing $s$ by $x$, that the spectral representation of the Mittag-Leffler function $E_{\nu}(-x)$ is expressed in terms of the $M$-Wright function $M_{\nu}(r)$, that is for $x \geq 0$

$E_{\nu}(-x)=\int_{0}^{\infty} \mathrm{e}^{-r x} M_{\nu}(r) d r, 0<\nu<1$.

We now recognize that Eq. (6.30) is consistent with a result derived by Pollard (1948) [51].

It is instructive to compare the spectral representation of $E_{\nu}(-x)$ with that of the function $E_{\nu}\left(-t^{\nu}\right)$. We recall for $t \geq 0$,

$E_{\nu}\left(-t^{\nu}\right)=\int_{0}^{\infty} \mathrm{e}^{-r t} K_{\nu}(r) d r, 0<\nu<1$,

with spectral function

$$
\begin{aligned}
K_{\nu}(r) & =\frac{1}{\pi} \frac{r^{\nu-1} \sin (\nu \pi)}{r^{2 \nu}+2 r^{\nu} \cos (\nu \pi)+1} \\
& =\frac{1}{\pi r} \frac{\sin (\nu \pi)}{r^{\nu}+r^{-\nu}+2 \cos (\nu \pi)} .
\end{aligned}
$$

The relationship between $M_{\nu}(r)$ and $K_{\nu}(r)$ is worth to be explored. Both functions are non-negative, integrable and normalized in $\mathbb{R}^{+}$, so they can be adopted in probability theory as density functions.
The transition $K_{\nu}(r) \rightarrow \delta(r-1)$ for $\nu \rightarrow 1$ is easy to be detected numerically in view of the explicit representation (6.32). On the contrary, the analogous transition $M_{\nu}(r) \rightarrow \delta(r-1)$ is quite a difficult matter in view of its series and integral representations. In this respect see the figure hereafter carried out in the 1997 paper by Mainardi and Tomirotti [42].
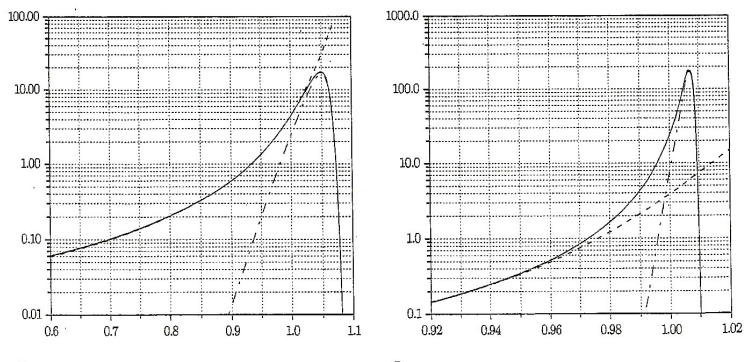

(a)

Figure 11: Plots of $M_{\nu}(r)$ with $\nu=1-\epsilon$ around the maximum $r \approx 1$.

Here we have compared the cases (a) $\epsilon=0.01$, (b) $\epsilon=0.001$, obtained by an asymptotic method originally due to Pipkin (continuous line), 100 terms-series (dashed line) and the standard saddle-point method (dashed-dotted line).

In the following Section we deal the asymptotic representations of the Wright functions for parameter $\lambda=-\nu$ not close to the singular case $\nu=1$.

\subsection{The Asymptotic representations}

For the asymptotic analysis in the whole complex plane for the Wright functions, the interested reader is referred to Wong and Zhao (1999a),(1999b)[61, 62], who have considered the two cases $\lambda \geq 0$ and $-1<$ $\lambda<0$ separately, including a description of Stokes' discontinuity and its smoothing.

For the Wright functions of the second kind, where $\lambda=-\nu \in(-1,0)$, we recall the asymptotic expansion originally obtained by Wright himself, that is valid in a suitable sector about the negative real axis as $|z| \rightarrow \infty$,

$$
\begin{aligned}
W_{-\nu, \mu}(z) & =Y^{1 / 2-\mu} \mathrm{e}^{-Y} \\
& \times\left[\sum_{m=0}^{M-1} A_{m} Y^{-m}+O\left(|Y|^{-M}\right)\right],
\end{aligned}
$$

with

$$
Y=Y(z)=(1-\nu)\left(-\nu^{\nu} z\right)^{1 /(1-\nu)},
$$

where the $A_{m}$ are certain real numbers. 
Let us first point out the asymptotic behaviour of the function $M_{\nu}(r)$ as $r \rightarrow \infty$. Choosing as a variable $r / \nu$ rather than $r$, the computation of the requested asymptotic representation by the saddle-point approximation yields, see Mainardi \& Tomirotti (1994) [41],

$$
\begin{aligned}
M_{\nu}(r / \nu) & \sim a(\nu) r^{(\nu-1 / 2) /(1-\nu)} \\
& \times \exp \left[-b(\nu) r^{1 /(1-\nu)}\right]
\end{aligned}
$$

where $a(\nu)$ and $b(\nu)$ are positive coefficients

$$
a(\nu)=\frac{1}{\sqrt{2 \pi(1-\nu)}}, b(\nu)=\frac{1-\nu}{\nu}>0 .
$$

The above evaluation is consistent with the first term in Wright's asymptotic expansion (6.33) after having used the definition (6.36).

We point out that in the limit $\nu \rightarrow 1^{-}$the function $M_{\nu}(r)$ tends to the Dirac function $\delta(r-1)$, but in a non-symmetric way as shown in the two plots in figure 11 of the previous subsection.

\section{The Wright function in Probability Theory}

Using the known completely monotone functions, the technique of the Laplace transform, and the Bernstein theorem, one can prove non-negativity of some Wright functions. Say, the function

$$
p_{\nu, \mu}(r)=\Gamma(\mu) W_{-\nu, \mu-\nu}(-r)
$$

can be interpreted as a one-sided probability density function (pdf) for $0<\nu \leq 1, \nu \leq \mu$ (see [27]). To show this, we use the Laplace transform pair (6.24) that we rewrite in the form

$$
W_{-\nu, \mu-\nu}(-r) \div E_{\nu, \mu}(-s), 0<\nu \leq 1,
$$

and the fact that the Mittag-Leffler function $E_{\nu, \mu}(-s)$ is completely monotone for $0<\nu \leq 1, \nu \leq \mu$. According to the Bernstein theorem, the function $p_{\nu, \mu}(r)$ is non-negative.

To calculate the integral of $p_{\nu, \mu}(r)$ over $\mathbb{R}^{+}$let us mention that it can be interpreted as the Laplace transform of $p_{\nu, \mu}$ at the point $s=0$ or the Mellin transform at $s=1$. Using the Mellin integral transform of the Wright function as in [26] leads now to the following chain of equalities:

$$
\begin{aligned}
\int_{0}^{\infty} p_{\nu, \mu}(r) d r & =\int_{0}^{\infty} \Gamma(\mu) W_{-\nu, \mu-\nu}(-r) d r \\
& =\left.\frac{\Gamma(\mu) \Gamma(s)}{\Gamma(\mu-\nu+\nu s)}\right|_{s=1}=\frac{\Gamma(\mu)}{\Gamma(\mu)}=1 .
\end{aligned}
$$

The Mellin transform technique allows us to calculate also all moments of order $s>0$ of the pdf $p_{\nu, \mu}(r)$ on $\mathbb{R}^{+}$:

$$
\begin{aligned}
\int_{0}^{\infty} p_{\nu, \mu}(r) r^{s} d r & =\int_{0}^{\infty} \Gamma(\mu) W_{-\nu, \mu-\nu}(-r) r^{s+1-1} d r \\
& =\frac{\Gamma(\mu) \Gamma(s+1)}{\Gamma(\mu+\nu s)} .
\end{aligned}
$$

For $\mu=1$, the pdf $p_{\nu, \mu}(r)$ can be expressed in terms of the $M$-Wright function $M_{\nu}(r), 0<\nu<1$ defined by Eq. (6.8). As it is well known (see, e.g., [32]), $M_{\nu}(r)$ can be interpreted as a one-sided pdf on $\mathbb{R}^{+}$ with the moments given by the formula

$$
\int_{0}^{\infty} M_{\nu}(r) r^{s} d r=\frac{\Gamma(s+1)}{\Gamma(1+\nu s)}, s>0 .
$$

\subsection{The Mainardi auxiliary functions as extremal stable densities}

We find it worthwhile to recall the relations between the Mainardi auxilary functions and the extremal Lévy stable densities as proven in the 1997 paper by Mainardi and Tomirotti [42]. For an essential account of the general theory of Lévy stable distributions in probability In the present paper the interested reader may consult the Appendix A in the present paper. More details can be found in the 1997 E-print by Mainardi-Gorenflo-Paradisi [40] and in the 2001 paper by Mainardi-Luchko-Pagnini. [36], recalled in the Appendix F in [32].

Then, from a comparison between the series expansions of stable densities according to the FekllerTakayasu canonic form with index of stability $\alpha \in$ $(0,2]$ and skewness $\theta(|\theta| \leq \min \{\alpha, 2-\alpha\})$, and those of the Mainardi auxiliary functions in Eqs. (6.7) - (6.8), we recognize, see also [33], that the auxiliary functions are related to the extremal stable densities as follows

$$
\begin{aligned}
L_{\alpha}^{-\alpha}(x) & =\frac{1}{x} F_{\alpha}\left(x^{-\alpha}\right)=\frac{\alpha}{x^{\alpha+1}} M_{\alpha}\left(x^{-\alpha}\right), \\
& 0<\alpha<1, \quad x \geq 0 . \\
L_{\alpha}^{\alpha-2}(x) & =\frac{1}{x} F_{1 / \alpha}(x)=\frac{1}{\alpha} M_{1 / \alpha}(x), \\
1 & <\alpha \leq 2, \quad-\infty<x<+\infty .
\end{aligned}
$$

In the above equations, for $\alpha=1$, the skewness parameter turns out to be $\theta=-1$, so we get the singular limit

$$
L_{1}^{-1}(x)=M_{1}(x)=\delta(x-1) .
$$

Hereafter we show the plots the extremal stable densities according to their expressions in terms of the $M$ Wright functions, see Eq. (7.1), Eq. (7.1) for $\alpha=1 / 2$ and $\alpha=3 / 2$, respectively. 


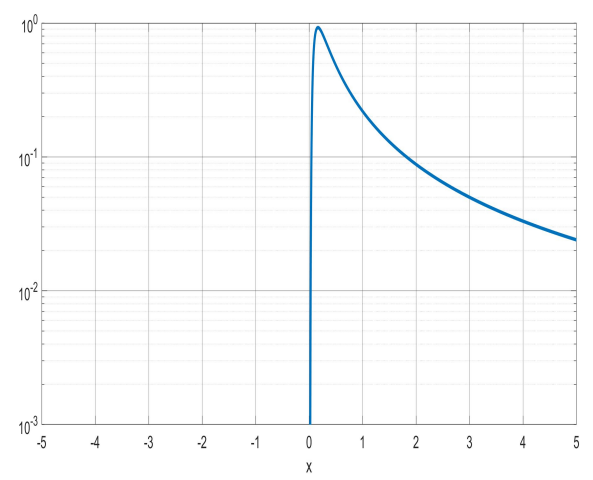

Figure 12: Plot of the unilateral extremal stable pdf for $\alpha=1 / 2$

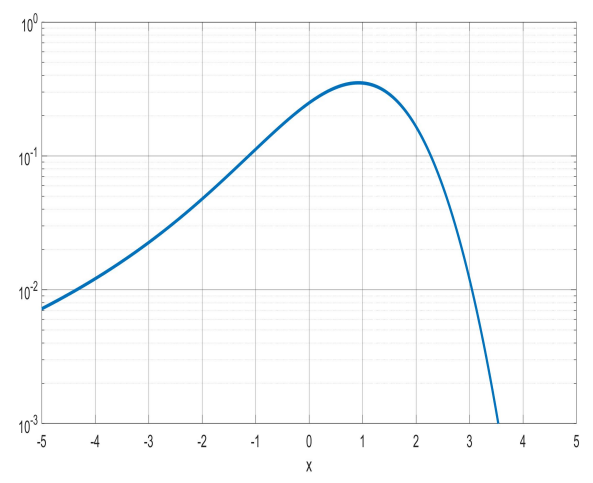

Figure 13: Plot of the bilateral extremal stable pdf for $\alpha=3 / 2$

\subsection{The plots and the Fourier transform of the symmetric M-Wright function}

We point out that the most relevant applications of our auxiliary functions, are when the variable is real. In particular we consider the case of the symmetric $M$ Wright function as a function of the variable $|x|$ for all $\mathbb{R}$ with varying its parameter $\nu \in[0,1]$ because related to the fundamental solution of the Cauchy problem of the time fractional diffusion-wave equation dealt in Appendix B In the following Figs. 14 and 15 we compare the plots of the $M_{\nu}(|x|)$-Wright functions in $|x| \leq 5$ for some rational values in the ranges $\nu \in[0,1 / 2]$ and $\nu \in[1 / 2,1]$, respectively. To gain more insight of the effect of the variation of the parameter $\nu$ we will adopt bot linear and $\log$ arithmic scales for the ordinate. Thus in Fig. 14 we see the transition from $\exp (-|x|)$ for $\nu=0$ to $1 / \sqrt{\pi} \exp \left(-x^{2}\right)$ for $\nu=1 / 2$, whereas in Fig. 15 we see the transition from $1 / \sqrt{\pi} \exp \left(-x^{2}\right)$ to the delta functions $\delta(x \pm 1)$ for $\nu=1$. In plotting $M_{\nu}(|x|)$ at fixed $\nu$ for sufficiently large $|x|$ the asymptotic representation (6.34)-(6.35) is useful since, as $|x|$ increases, the numerical convergence of the series in (6.11) becomes poor and poor up to being completely inefficient: henceforth, the matching between the series and the asymptotic representation is relevant. However, as $\nu \rightarrow 1^{-}$, the plotting remains a very difficult task because of the high peak arising around $x= \pm 1$.
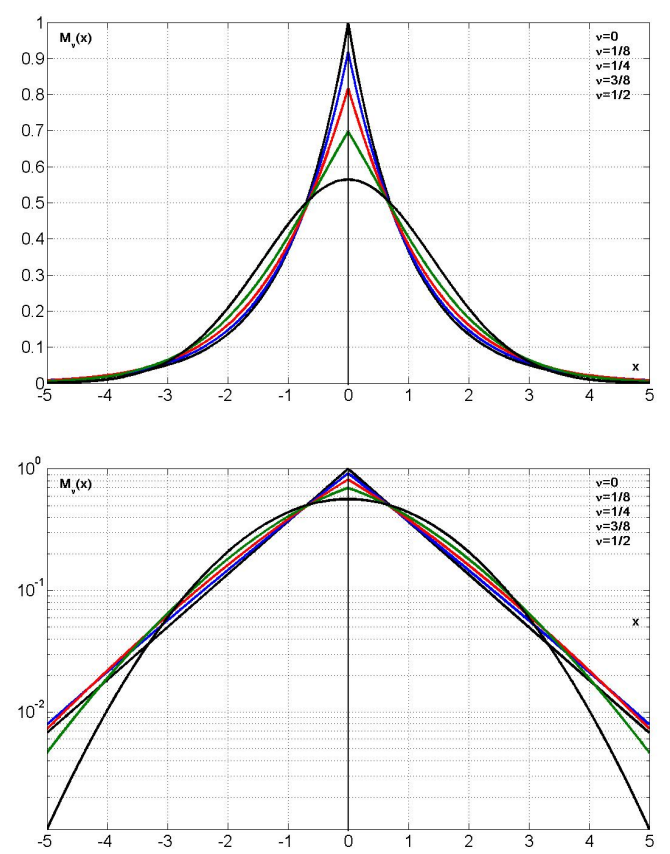

Figure 14: Plots of $M_{\nu}(|x|)$ with $\nu=$ $0,1 / 8,1 / 4,3 / 8,1 / 2$ for $|x| \leq 5$; top: linear scale, bottom: logarithmic scale.

The Fourier transform of the $M$-Wright function. The Fourier transform of the symmetric (and normalized) $M$-Wright function provides its characteristic function useful in Probability theory.

$$
\begin{aligned}
& \mathcal{F}\left[\frac{1}{2} M_{\nu}(|x|)\right] \equiv \frac{1}{2} \widehat{M_{\nu}(|x|)} \\
& :=\frac{1}{2} \int_{-\infty}^{+\infty} \mathrm{e}^{i \kappa x} M_{\nu}(|x|) d x \\
& =\int_{0}^{\infty} \cos (\kappa x) M_{\nu}(x) d x=E_{2 \nu}\left(-\kappa^{2}\right) .
\end{aligned}
$$

For this prove it is sufficient to develop in series the cosine function and use the formula for the absolute moments of the $M$-Wright function in $\mathbb{R}^{+}$.

$$
\begin{aligned}
& \int_{0}^{\infty} \cos (\kappa x) M_{\nu}(x) d x \\
& =\sum_{n=0}^{\infty}(-1)^{n} \frac{\kappa^{2 n}}{(2 n) !} \int_{0}^{\infty} x^{2 n} M_{\nu}(x) d x \\
& =\sum_{n=0}^{\infty}(-1)^{n} \frac{\kappa^{2 n}}{\Gamma(2 \nu n+1)}=E_{2 \nu, 1}\left(-\kappa^{2}\right) .
\end{aligned}
$$



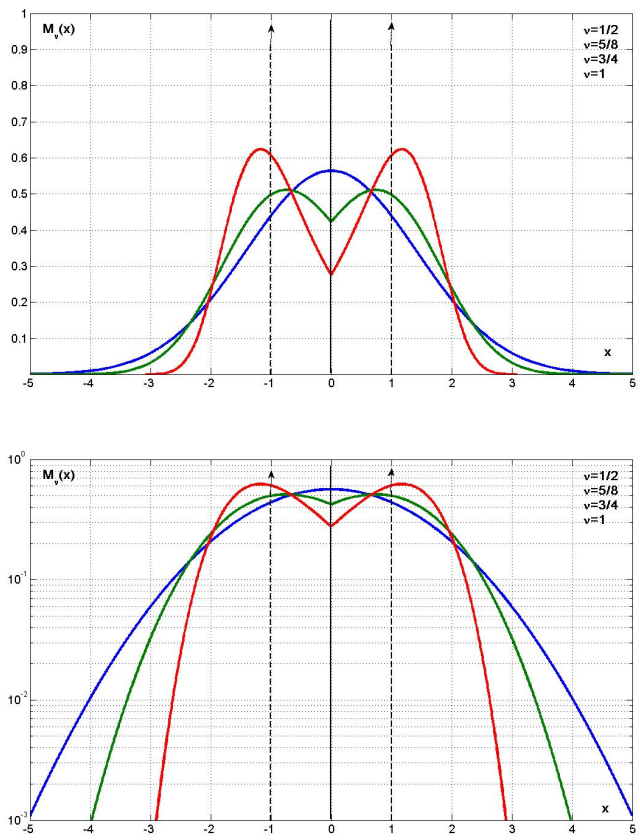

Figure 15: Plots of $M_{\nu}(|x|)$ with $\nu=$ $1 / 2,5 / 8,3 / 4,1$ for $|x| \leq 5$ : top: linear scale; bottom: logarithmic scale)

We also have

$$
\begin{aligned}
& \int_{0}^{\infty} \sin (\kappa x) M_{\nu}(x) d x \\
& =\sum_{n=0}^{\infty}(-1)^{n} \frac{\kappa^{2 n+1}}{(2 n+1) !} \int_{0}^{\infty} x^{2 n+1} M_{\nu}(x) d x \\
& =\sum_{n=0}^{\infty}(-1)^{n} \frac{\kappa^{2 n+1}}{\Gamma(2 \nu n+1+\nu)}=\kappa E_{2 \nu, 1+\nu}\left(-\kappa^{2}\right) .
\end{aligned}
$$

\subsection{Subordination formulas}

We now consider $M$-Wright functions as spatial probability densities evolving in time with self-similarity, that is

$$
M_{\nu}(x, t):=t^{-\nu} M_{\nu}\left(x t^{-\nu}\right), x, t \geq 0 .
$$

These $M$-Wright functions are relevant for their composition rules proved by Mainardi et al. in [36], and more generally in [38] by using the Mellin Transforms.

The main statement can be summarized as: Let $M_{\lambda}(x ; t), M_{\mu}(x ; t)$ and $M_{\nu}(x ; t)$ be $M$-Wright functions of orders $\lambda, \mu, \nu \in(0,1)$ respectively, then the following composition formula holds for any

$$
\begin{aligned}
& x, t \geq 0: \\
& M_{\nu}(x, t)=\int_{0}^{\infty} M_{\lambda}(x ; \tau) M_{\mu}(\tau ; t) d \tau, \nu=\lambda \mu .
\end{aligned}
$$

The above equation is also intended as a subordination formula because it can be used to define subordination among self-similar stochastic processes (with independent increments), that properly generalize the most popular Gaussian processes, to which they reduce for $\nu=1 / 2$.

These more general processes are governed by timefractional diffusion equations, as shown in papers of our research group, see Mura-Pagnini (JPhysA 2008), Mura-Taqqu-Mainardi (PhysicaA 2008). MuraMainardi (ITSF 2009) These general processes are referred to as Generalized grey Brownian Motions, that include both Gaussian Processes (standard Brownian motion, fractional Brownian motion) and nonGaussian Processes (Schneider's grey Brownian motion), to which the interested reader is referred for details.

\section{Conclusions}

In this survey we have outlined the basic properties of the Mittag-Leffler and Wright functions. We have also considered a number of applications taking into account special functions of these families. We have stressed their relations with fractional calculus. In particular, we have added a number of tutorial appendices to enlarge the fields of applicability of the Wright functions, nowadays less known than the Mittag-Leffler functions to which they are related through integral transforms.

\section{Acknowledgments}

The research activity of the author is carried out in the framework of the activities of the National Group of Mathematical Physics (GNFM, INdAM), Italy.

\section{Appendix A: Lévy stable distributions}

The term stable has been assigned by the French mathematician Paul Lévy, who in the 1920's years started a systematic research in order to generalize the celebrated Central Limit Theorem to probability distributions with infinite variance. For stable distributions we can assume the following 
DEFINITION: If two independent real random variables with the same shape or type of distribution are combined linearly and the distribution of the resulting random variable has the same shape, the common distribution (or its type, more precisely) is said to be stable.

The restrictive condition of stability enabled Lévy (and then other authors) to derive the canonic form for the Fourier transform of the densities of these distributions. Such transform in probability theory is known as characteristic function.

Here we follow the parameterization adopted in Feller (1971) [11] revisited in 1998 by Gorenflo \& Mainardi [20] and popularized in 2001 by Mainardi, Luchko \& Pagnini [36].

Denoting by $L_{\alpha}^{\theta}(x)$ a (strictly) stable density in $\mathbb{R}$, where $\alpha$ is the index of stability and an $\theta$ the asymmetry parameter, improperly called skewness, its characteristic function reads:

$$
\begin{aligned}
& L_{\alpha}^{\theta}(x) \div \widehat{L}_{\alpha}^{\theta}(\kappa)=\exp \left[-\psi_{\alpha}^{\theta}(\kappa)\right], \\
& \psi_{\alpha}^{\theta}(\kappa)=|\kappa|^{\alpha} \mathrm{e}^{i(\operatorname{sign} \kappa) \theta \pi / 2},
\end{aligned}
$$

with

$$
0<\alpha \leq 2,|\theta| \leq \min \{\alpha, 2-\alpha\} .
$$

We note that the allowed region for the parameters $\alpha$ and $\theta$ turns out to be a diamond in the plane $\{\alpha, \theta\}$ with vertices in the points $(0,0),(1,1),(1,-1)$, $(2,0)$, that we call the Feller-Takayasu diamond, see Fig.16. For values of $\theta$ on the border of the diamond (that is $\theta= \pm \alpha$ if $0<\alpha<1$, and $\theta= \pm(2-\alpha)$ if $1<\alpha<2$ ) we obtain the so-called extremal stable densities.

We note the symmetry relation $L_{\alpha}^{\theta}(-x)=L_{\alpha}^{-\theta}(x)$, so that a stable density with $\theta=0$ is symmetric

Stable distributions have noteworthy properties of which the interested reader can be informed from the existing literature. Here-after we recall some peculiar Properties:

- The class of stable distributions possesses its own domain of attraction, see e.g. Feller (1971)[11].

- Any stable density is unimodal and indeed bellshaped, i.e. its $n$-th derivative has exactly $n$ zeros in $\mathbb{R}$, see Gawronski (1984) [14] and Simon (2015) [56].

- The stable distributions are self-similar and infinitely divisible. These properties derive from the canonic form (A.1)-(A.2) through the scaling property of the Fourier transform.

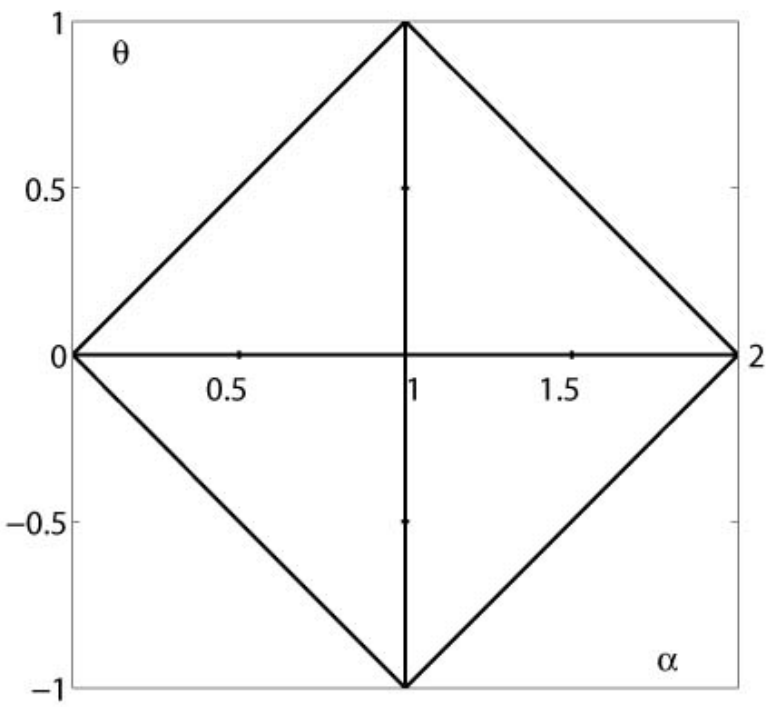

Figure 16: The Feller-Takayasu diamond for Lévy stable densities.

Self-similarity means

$$
\begin{aligned}
& L_{\alpha}^{\theta}(x, t) \div \exp \left[-t \psi_{\alpha}^{\theta}(\kappa)\right] \\
& \left.\Longleftrightarrow L_{\alpha}^{\theta}(x, t)=t^{-1 / \alpha} L_{\alpha}^{\theta}\left(x / t^{1 / \alpha}\right)\right],
\end{aligned}
$$

where $t$ is a positive parameter. If $t$ is time, then $L_{\alpha}^{\theta}(x, t)$ is a spatial density evolving on time with self-similarity.

Infinite divisibility means that for every positive integer $n$, the characteristic function can be expressed as the $n$th power of some characteristic function, so that any stable distribution can be expressed as the $n$-fold convolution of a stable distribution of the same type. Indeed, taking in (A.3) $\theta=0$, without loss of generality, we have

$$
\begin{aligned}
\mathrm{e}^{-t|\kappa|^{\alpha}} & =\cdot\left[\mathrm{e}^{-(t / n)|\kappa|^{\alpha}}\right]^{n} \\
& \Longleftrightarrow L_{\alpha}^{0}(x, t)=\left[L_{\alpha}^{0}(x, t / n)\right]^{* n},
\end{aligned}
$$

where

$$
\left[L_{\alpha}^{0}(x, t / n)\right]^{* n}:=L_{\alpha}^{0}(x, t / n) * \ldots * L_{\alpha}^{0}(x, t / n)
$$

is the multiple Fourier convolution in $\mathbb{R}$ with $n$ identical terms.

Only in special cases we get well-known probability distributions.

For $\alpha=2$ (so $\theta=0$ ), we recover the Gaussian $p d f$, that turns out to be the only stable density with finite variance, and more generally with finite moments of any order $\delta \geq 0$. In fact

$$
L_{2}^{0}(x)=\frac{1}{2 \sqrt{\pi}} \mathrm{e}^{-x^{2} / 4} .
$$


All the other stable densities have finite absolute moments of order $\delta \in[-1, \alpha)$.

For $\alpha=1$ and $|\theta|<1$, we get

$L_{1}^{\theta}(x)=\frac{1}{\pi} \frac{\cos (\theta \pi / 2)}{[x+\sin (\theta \pi / 2)]^{2}+[\cos (\theta \pi / 2)]^{2}}$,

which for $\theta=0$ includes the Cauchy-Lorentz $p d f$,

$$
L_{1}^{0}(x)=\frac{1}{\pi} \frac{1}{1+x^{2}}
$$

In the limiting cases $\theta= \pm 1$ for $\alpha=1$ we obtain the singular Dirac pdf's

$$
L_{1}^{ \pm 1}(x)=\delta(x \pm 1) .
$$

In general we must recall the power series expansions provided by Feller (1971) [11]. We restrict our attention to $x>0$ since the evaluations for $x<0$ can be obtained using the symmetry relation.

The convergent expansions of $L_{\alpha}^{\theta}(x)(x>0)$ turn out to be

for $0<\alpha<1,|\theta| \leq \alpha$ :

$L_{\alpha}^{\theta}(x)=\frac{1}{\pi x} \sum_{n=1}^{\infty}\left(-x^{-\alpha}\right)^{n} \frac{\Gamma(1+n \alpha)}{n !} \sin \left[\frac{n \pi}{2}(\theta-\alpha)\right]$,

for $1<\alpha \leq 2,|\theta| \leq 2-\alpha$ :

$L_{\alpha}^{\theta}(x)=\frac{1}{\pi x} \sum_{n=1}^{\infty}(-x)^{n} \frac{\Gamma(1+n / \alpha)}{n !} \sin \left[\frac{n \pi}{2 \alpha}(\theta-\alpha)\right]$.

From the series (A.9) and the symmetry relation we note that the extremal stable densities for $0<\alpha<1$ are unilateral, precisely vanishing for $x>0$ if $\theta=\alpha$, vanishing for $x<0$ if $\theta=-\alpha$. In particular the unilateral extremal densities $L_{\alpha}^{-\alpha}(x)$ with $0<\alpha<1$ have as Laplace transform $\exp \left(-s^{\alpha}\right)$.

From a comparison between the series expansions in (A.9)-(A.10) and in (6.10)-(6.11) for the auxiliary functions $F_{\alpha}(x), M_{\alpha}(x)$ we recognize that for $x>0$ the auxiliary functions of the Wright type are related to the extremal stable densities as in Eqs. (6.28)(6.29).

More generally, all (regular) stable densities, given in Eqs. (6.38)-(6.39), were recognized to belong to the class of Fox $H$-functions, as formerly shown in 1986 by Schneider [55], see also Mainardi-Pagnini-Saxena (2003) [39].

\section{Appendix B: The time-fractional diffusion equation}

There exist three equivalent forms of the timefractional diffusion equation of a single order, two with fractional derivative and one with fractional integral, provided we refer to the standard initial condition $u(x, 0)=u_{0}(x)$.

Taking a real number $\beta \in(0,1)$, the time-fractional diffusion equation of order $\beta$ in the RiemannLiouville sense reads

$$
\frac{\partial u}{\partial t}=K_{\beta} D_{t}^{1-\beta} \frac{\partial^{2} u}{\partial x^{2}},
$$

in the Caputo sense reads

$$
{ }_{*} D_{t}^{\beta} u=K_{\beta} \frac{\partial^{2} u}{\partial x^{2}},
$$

and in integral form

$u(x, t)=u_{0}(x)+K_{\beta} \frac{1}{\Gamma(\beta)} \int_{0}^{t}(t-\tau)^{\beta-1} \frac{\partial^{2} u(x, \tau)}{\partial x^{2}} d \tau$.

where $K_{\beta}$ is a sort of fractional diffusion coefficient of dimensions $\left[K_{\beta}\right]=[L]^{2}[T]^{-\beta}=\mathrm{cm}^{2} / \mathrm{sec}^{\beta}$.

The fundamental solution (or Green function) $\mathcal{G}_{\beta}(x, t)$ for the equivalent Eqs. (B.1) - (B.3), that is the solution corresponding to the initial condition

$$
\mathcal{G}_{\beta}\left(x, 0^{+}\right)=u_{0}(x)=\delta(x)
$$

can be expressed in terms of the $M$-Wright function

$$
\mathcal{G}_{\beta}(x, t)=\frac{1}{2} \frac{1}{\sqrt{K_{\beta}} t^{\beta / 2}} M_{\beta / 2}\left(\frac{|x|}{\sqrt{K_{\beta}} t^{\beta / 2}}\right) .
$$

The corresponding variance can be promptly obtained

$$
\sigma_{\beta}^{2}(t):=\int_{-\infty}^{+\infty} x^{2} \mathcal{G}_{\beta}(x, t) d x=\frac{2}{\Gamma(\beta+1)} K_{\beta} t^{\beta} .
$$

As a consequence, for $0<\beta<1$ the variance is consistent with a process of slow diffusion with similarity exponent $H=\beta / 2$.

The fundamental solution $\mathcal{G}_{\beta}(x, t)$ for the timefractional diffusion equation can be obtained by applying in sequence the Fourier and Laplace transforms to any form chosen among Eqs. (B.1)-(B.3). Let us devote our attention to the integral form (B.3) using non-dimensional variables by setting $K_{\beta}=1$ and 
adopting the notation $J_{t}^{\beta}$ for the fractional integral. Then, our Cauchy problem reads

$$
\mathcal{G}_{\beta}(x, t)=\delta(x)+J_{t}^{\beta} \frac{\partial^{2} \mathcal{G}_{\beta}}{\partial x^{2}}(x, t) .
$$

In the Fourier-Laplace domain, after applying formula for the Laplace transform of the fractional integral and observing $\widehat{\delta}(\kappa) \equiv 1$, we get

$$
\widehat{\mathcal{G}_{\beta}}(\kappa, s)=\frac{1}{s}-\frac{\kappa^{2}}{s^{\beta}} \widehat{\widetilde{\mathcal{G}_{\beta}}}(\kappa, s),
$$

from which for $\operatorname{Re}(s)>0, \kappa \in \mathbb{R}$

$$
\widehat{\widehat{\mathcal{G}}}(\kappa, s)=\frac{s^{\beta-1}}{s^{\beta}+\kappa^{2}}, \quad 0<\beta \leq 1 . .
$$

Strategy (S1): Recalling the Fourier transform pair

$$
\frac{a}{b+\kappa^{2}} \div \frac{a}{2 b^{1 / 2}} \mathrm{e}^{-|x| b^{1 / 2}}, a, b>0,
$$

and setting $a=s^{\beta-1}, b=s^{\beta}$, we get

$$
\widetilde{\mathcal{G}_{\beta}}(x, s)=\frac{1}{2} s^{\beta / 2-1} \mathrm{e}^{-|x| s^{\beta / 2}} .
$$

Strategy (S2): Recalling the Laplace transform pair

$$
\frac{s^{\beta-1}}{s^{\beta}+c} \stackrel{\mathcal{L}}{\leftrightarrow} E_{\beta}\left(-c t^{\beta}\right), c>0,
$$

and setting $c=\kappa^{2}$, we have

$$
\widehat{\mathcal{G}_{\beta}}(\kappa, t)=E_{\beta}\left(-\kappa^{2} t^{\beta}\right) .
$$

Both strategies lead to the result

$\mathcal{G}_{\beta}(x, t)=\frac{1}{2} M_{\beta / 2}(|x|, t)=\frac{1}{2} t^{-\beta / 2} M_{\beta / 2}\left(\frac{|x|}{t^{\beta / 2}}\right)$,

consistent with Eq. (B.5).

\section{The time-fractional drift equation}

Let us finally note that the $M$-Wright function does appear also in the fundamental solution of the timefractional drift equation. Writing this equation in nondimensional form and adopting the Caputo derivative we have

${ }_{*} D_{t}^{\beta} u(x, t)=-\frac{\partial}{\partial x} u(x, t), \quad-\infty<x<+\infty, t \geq 0$,

where $0<\beta<1$ and $u\left(x, 0^{+}\right)=u_{0}(x)$. When $u_{0}(x)=\delta(x)$ we obtain the fundamental solution
(Green function) that we denote by $\mathcal{G}_{\beta}^{*}(x, t)$. Following the usual approach we show that

$$
\mathcal{G}_{\beta}^{*}(x, t)= \begin{cases}t^{-\beta} M_{\beta}\left(\frac{x}{t^{\beta}}\right), & x>0, \\ 0, & x<0\end{cases}
$$

that for $\beta=1$ reduces to the right running pulse $\delta(x-$ t) for $x>0$.

In the Fourier-Laplace domain, after applying the formula for the Laplace transform of the Caputo fractional derivative and observing $\widehat{\delta}(\kappa) \equiv 1$, we get

$$
s^{\beta} \widehat{\widehat{\mathcal{G}_{\beta}^{*}}}(\kappa, s)-s^{\beta-1}=+i \kappa \widehat{\widehat{\mathcal{G}_{\beta}^{*}}}(\kappa, s),
$$

from which, for $\operatorname{Re}(s)>0, \kappa \in \mathbb{R}$

$$
\widehat{\widehat{\mathcal{G}}}(\kappa, s)=\frac{s^{\beta-1}}{s^{\beta}-i \kappa}, 0<\beta \leq 1,
$$

To determine the Green function $\mathcal{G}_{\beta}^{*}(x, t)$ in the spacetime domain we can follow two alternative strategies related to the order in carrying out the inversions in (B.16).

(S1) : invert the Fourier transform getting $\widetilde{\mathcal{G}_{\beta}}(x, s)$ and then invert the remaining Laplace transform;

(S2) : invert the Laplace transform getting $\widehat{\mathcal{G}_{\beta}^{*}}(\kappa, t)$ and then invert the remaining Fourier transform. Strategy (S1): Recalling the Fourier transform pair

$$
\frac{a}{b-i \kappa} \stackrel{\mathcal{F}}{\leftrightarrow} \frac{a}{b} \mathrm{e}^{-x b}, a, b>0, x>0,
$$

and setting $a=s^{\beta-1}, b=s^{\beta}$, we get

$$
\widetilde{\mathcal{G}_{\beta}^{*}}(x, s)=s^{\beta-1} \mathrm{e}^{-x s^{\beta}} .
$$

Strategy (S2): Recalling the Laplace transform pair

$$
\frac{s^{\beta-1}}{s^{\beta}+c} \stackrel{\mathcal{L}}{\leftrightarrow} E_{\beta}\left(-c t^{\beta}\right), \quad c>0,
$$

and setting $c=-i \kappa$, we have

$$
\widehat{\mathcal{G}_{\beta}^{*}}(\kappa, t)=E_{\beta}\left(i \kappa t^{\beta}\right) .
$$

Both strategies lead to the result (B.15). In view of Eq. (7.5) we also recall that the $M$-Wright function is related to the unilateral extremal stable density of index $\beta$. Then, using our notation for stable densities, we write our Green function (B.15) as

$$
\mathcal{G}_{\beta}^{*}(x, t)=\frac{t}{\beta} x^{-1-1 / \beta} L_{\beta}^{-\beta}\left(t x^{-1 / \beta}\right) .
$$




\section{Appendix C: Historical and bibliographic notes}

In the early nineties, precisely in his 1993 former analysis of fractional equations describing slow diffusion and interpolating diffusion and wave-propagation, the present author [28], introduced the so called auxiliary functions of the Wright type $F_{\nu}(z):=W_{-\nu, 0}(-z)$ and $M_{\nu}(z):=W_{-\nu, 1-\nu}(-z)$ with $0<\nu<1$, in order to characterize the fundamental solutions for typical boundary value problems, as it is shown in the previous sections. Being then only aware of the Handbook of the Bateman project, where the parameter $\lambda$ of the Wright function $W_{\lambda, \mu}(z)$ was erroneously restricted to non-negative values, the author thought to have originally extended the analyticity property of the original Wright function by taking $\nu=-\lambda$ with $\nu \in(0,1)$. Then the function $M_{\nu}$ was referred to as the Mainardi function in the 1999 treatise by Podlubny [49] and in some later papers and books.

It was Professor B. Stanković during the presentation of the paper by Mainardi \& Tomirotti [41] at the Conference Transform Methods and Special Functions, Sofia 1994, who informed the author that this extension for $-1<\lambda<0$ had been already made by Wright himself in 1940 (following his previous papers in the thirties), see [65]. In a 2005 paper published in FCAA [35], devoted to the 80th birthday of Professor Stanković, the author used the occasion to renew his personal gratitude to Professor Stanković for this earlier information that led him to study the original papers by Wright and to work (also in collaboration) on the functions of the Wright type for further applications, see e.g. [16, 17] and [37]. For the above reasons the author preferred to distinguish the Wright functions into the first kind $(\lambda \geq 0)$ and the second kind $(-1<\lambda<0)$.

It should be noted that in the book by Prüss (1993) [52] we find a figure quite similar to our Fig 15-top reporting the $M$-Wright function in linear scale, namely the Wright function of the second kind. It was derived from inverting the Fourier transform expressed in terms of the Mittag-Leffler function following the approach by Fujita [12] for the fundamental solution of the Cauchy problem for the diffusion-wave equation, fractional in time. However, our plot must be considered independent by that of Prüss because the author (Mainardi) used the Laplace transform in his former paper presented at WASCOM, Bologna, October 1993 [28] (and published later in a number of papers and in his 2010 book) so he was aware of the book by Prüss only later.

\section{References:}

[1] J.H. Barret (1954). Differential equations of noninteger order, Canad. J. Math 6, 529-541.

[2] P.W. Buchen and F. Mainardi (1975). Asymptotic expansions for transient viscoelastic waves, Journal de Mécanique 14, 597-608.

[3] M. Caputo and F. Mainardi (1971a). A new dissipation model based on memory mechanism, Pure and Appl. Geophys. (PAGEOPH) 91, 134-147. [Reprinted in Fract. Calc. Appl. Anal. 10 No 3, 309324 (2007)]

[4] M. Caputo and F. Mainardi (1971b). Linear models of dissipation in anelastic solids, Riv. Nuovo Cimento (Ser. II) 1, 161-198.

[5] K.S. Cole (1933). Electrical conductance of biological systems, Electrical excitation in nerves, in Proceedings Symposium on Quantitative Biololgy, Cold Spring Harbor, New York, Vol. 1, pp. 107-116.

[6] A. Consiglio \& F. Mainardi (2019). On the evolution of fractional diffusive waves. Ricerche $d i$ Matematica, published on line 06 Dec. 2019. DOI: $\quad 10.1007 / \mathrm{s} 11587-019-00476-6 \quad$ [E-print: arxiv.org/abs/1910.1259]

[7] H.T. Davis (1936). The Theory of Linear Operators, The Principia Press, Bloomington, Indiana.

[8] G. Doetsch (1974). Introduction to the Theory and Application of the Laplace Transformation, Springer Verlag, Berlin.

[9] M.M. Dzherbashyan (1966). Integral Transforms and Representations of Functions in the Complex Plane, Nauka, Moscow [in Russian]. There is also the transliteration as Djrbashyan.

[10] A. Erdélyi, W. Magnus, F. Oberhettinger and F. Tricomi, (1955). Higher Transcendental Functions, 3rd Volume, McGraw-Hill, New York . [Bateman Project].

[11] W. Feller (1971). An Introduction to Probability Theory and its Applications. Wiley, New York, Vol. II, Second Edition.

[12] Y. Fujita (1990). Integro-differential equation which interpolates the heat equation and the wave equation, I, II, Osaka J. Math. 27, 309-321, 797-804.

[13] L.G. Gatteschi (1973). Funzioni Speciali, UTET, Torino.

[14] W. Gawronski (1984). On the bell-shape of stable distributions, Annals of Probability 12, 230-242. 
[15] R. Gorenflo, A.A. Kilbas, F. Mainardi \& S. Rogosin (2014). Mittag-Leffler Functions. Related Topics and Applications, Springer, Berlin. Second Edition in preparation.

[16] R. Gorenflo, Yu. Luchko and F. Mainardi (1999). Analytical properties and applications of the Wright function. Fract. Calc. Appl. Anal. 2, 383-414.

[17] R. Gorenflo, Yu. Luchko and F. Mainardi (2000). Wright functions as scale-invariant solutions of the diffusion-wave equation, Journal of Computational and Applied Mathematics 118, 175-191.

[18] R. Gorenflo and F. Mainardi (1996). Fractional oscillations and Mittag-Leffler functions, Preprint No A-96-14, Fachbereich Mathematik und Informatik, Freie Universität Berlin, Serie Mathematik, pp. 22. [E-print: www.math. fu-berlin.de/publ/index.html]

[19] R. Gorenflo and F. Mainardi (1997). Fractional calculus: integral and differential equations of fractional order, in: A. Carpinteri and F. Mainardi (Editors): Fractals and Fractional Calculus in Continuum Mechanics, Springer Verlag, Wien, pp. 223-276. [Eprint: http://arxiv.org/abs/0805.3823]

[20] R. Gorenflo and F. Mainardi (1998). Random walk models for space-fractional diffusion processes, Fract. Calc. Appl. Anal. 1 No 2, 167-190.

[21] B. Gross (1947). On creep and relaxation, J. Appl. Phys. 18, 212-221.

[22] E. Hille and J.D. Tamarkin (1930). On the theory of linear integral equations, Ann. Math. 31, 479-528.

[23] V. Kiryakova (1994). Generalized Fractional Calculus and Applications, Longman \& J. Wiley, Harlow New York. [Pitman Research Notes in Mathematics, Vol. 301]

[24] A. Liemert and A. Klenie (2015). Fundamental Solution of the Tempered Fractional Diffusion Equation. J. Math. Phys 56, 113504.

[25] Yu. Luchko (2000). On the asymptotics of zeros of the Wright function. Zeitschrift für Analysis und ihre Anwendungen, 19, 597-622.

[26] Yu. Luchko (2019). The Wright function and its applications, in A. Kochubei, Yu.Luchko (Eds.), Handbook of Fractional Calculus with Applications Vol. 1: Basic Theory, pp. 241- 268. De Gruyter GmbH, 2019, Berlin/Boston. Series edited by J. A.Tenreiro Machado.

[27] Yu. Luchko \& V. Kiryakova (2013). The Mellin integral transform in fractional calculus, Fract. Calc. Appl. Anal. 16, 405-430.
[28] F. Mainardi (1994). On the initial value problem for the fractional diffusion-wave equation, in: Rionero, S. and Ruggeri, T. (Editors), Waves and Stability in Continuous Media. World Scientific, Singapore, pp. 246-251. [Proc. VII-th WASCOM, Int. Conf. "Waves and Stability in Continuous Media", Bologna, Italy, 4-7 October 1993]

[29] F. Mainardi (1996a). The fundamental solutions for the fractional diffusion-wave equation. Applied Mathematics Letters,9 No 6, 23-28.

[30] F. Mainardi (1996b). Fractional relaxationoscillation and fractional diffusion-wave phenomena. Chaos, Solitons \& Fractals 7, 1461-1477.

[31] F. Mainardi (1997). Fractional calculus: some basic problems in continuum and statistical mechanics, in: A. Carpinteri and F. Mainardi (Editors): Fractals and Fractional Calculus in Continuum Mechanics, Springer Verlag, Wien, pp. 291-348. [E-print: http://arxiv.org/abs/1201.0863]

[32] F. Mainardi (2010). Fractional Calculus and Waves in Linear Viscoelasticity. Imperial College Press, London and World Scientific, Singapore.

[33] F. Mainardi and A. Consiglio (2020). The Wright functions of the second kind in Mathematical Physics. PRE-PRINT submitted to Mathematics.

[34] F. Mainardi and R. Gorenflo (2007). Time-fractional derivatives in relaxation processes: a tutorial survey, Fract. Calc. Appl. Anal. 10, 269-308. [E-print: http://arxiv.org/abs/0801.4914]

[35] F. Mainardi, R. Gorenflo and A. Vivoli (2005). Renewal processes of Mittag-Leffler and Wright type, Fract. Calc. Appl. Anal. 8, 7-38. [E-print http://arxiv.org/abs/math/0701455]

[36] F. Mainardi, Yu. Luchko and G. Pagnini (2001). The fundamental solution of the spacetime fractional diffusion equation. Fract. Calc. Appl. Anal. 4, 153-192. [E-print: arxiv.org/abs/cond-mat/0702419]

[37] F. Mainardi and G. Pagnini (2003). The Wright functions as solutions of the time-fractional diffusion equation. Applied Mathematics and Computation 141 No 1, 51-62.

[38] F. Mainardi, G. Pagnini and R. Gorenflo (2003). Mellin transform and subordination laws in fractional diffusion processes, Fract. Calc. Appl. Anal. 6 No 4, 441-459. [E-print: http://arxiv.org/abs/math/0702133]

[39] F. Mainardi, G. Pagnini and R.K. Saxena (2005). Fox $H$ functions in fractional diffusion, J. Comp. Appl. Math. 178, 321-331. 
[40] F. Mainardi, P. Paradisi and R. Gorenflo (1998), Probability distributions generated by fractional diffusion equations, Invited Lecture, Workshop on Econophysics, Budapest 21-27 July 1997. LaTeX Pre-print, Dept. of Physics, Bologna, January 1998, pp. ii +39. [E-print http://arxiv.org/abs/0704.0320] N.B. It would have appeared in J. Kertesz and I. Kondor (Editors), Econophysics: an Emerging Science, Kluwer, Dordrecht, book NOT published!

[41] F. Mainardi and M. Tomirotti (1995). On a special function arising in the time fractional diffusion-wave equation, in: P. Rusev, I. Dimovski and V. Kiryakova, (Editors), Transform Methods and Special Functions, Sofia 1994, Science Culture Technology, Singapore, pp. 171-183.

[42] F. Mainardi and M. Tomirotti (1997). Seismic Pulse Propagation with Constant $Q$ and Stable Probability Distributions. Annali di Geofisica 40, 1311-1328. [Eprint: arxiv.org/abs/1008.1341]

[43] J. Mikusiński (1959). On the function whose Laplace transform is $\exp \left(-s^{\alpha}\right)$, Studia Math. 18, 191-198.

[44] G.M. Mittag-Leffler (1903a). Une généralisation de l'intégrale de Laplace-Abel, C.R. Acad. Sci. Paris (Ser. II) 137, 537-539.

[45] G.M. Mittag-Leffler (1903b). Sur la nouvelle fonction $E_{\alpha}(x)$, C.R. Acad. Sci. Paris (Ser. II) 137, 554 558.

[46] G.M. Mittag-Leffler (1904). Sopra la funzione $E_{\alpha}(x)$, Rendiconti R. Accademia Lincei (Ser. V) 13, 3-5.

[47] G.M. Mittag-Leffler (1905). Sur la représentation analytique d'une branche uniforme d'une fonction monogène, Acta Math. 29, 101-181.

[48] R.B. Paris (2019). Asymptotics of the special functions of fractional calculus, in: A. Kochubei, Yu.Luchko (Editors), Handbook of Fractional Calculus with Applications, Vol. 1: Basic Theory, pp. 297325. De Gruyter GmbH, 2019 Berlin/Boston. Series edited by J. A.Tenreiro Machado.

[49] I. Podlubny (1999). Fractional Differential Equations, Academic Press, San Diego.

[50] H. Pollard (1946). The representation of $\exp \left(-x^{\lambda}\right)$ as a Laplace integral, Bull. Amer. Math. Soc. 52, 908910 .

[51] H. Pollard, (1948). The completely monotonic character of the Mittag-Leffler function $E_{\alpha}(-x)$, Bull. Amer. Math. Soc. 54, 1115-1116
[52] J. Pr"uss, (1993). Evolutionary Integral Equations and Applications. Birkhauser Verlag, Basel.

[53] A. Saa and R. Venegeroles (2011). Alternative numerical computation of one-sided Lévy and MittagLeffler distributions, Phys. Rev. E 84, 026702.

[54] G. Sansone and J. Gerretsen. (1960). Lectures on the Theory of Functions of a Complex Variable, Vol. I. Holomorphic Functions, Nordhoff, Groningen.

[55] W.R. Schneider (1986). Stable distributions: Fox function representation and generalization, in S. Albeverio, G. Casati and D. Merlini, D. (Editors), Stochastic Processes in Classical and Quantum Systems, Springer Verlag, Berlin, pp. 497-511. [Lecture Notes in Physics, Vol. 262]

[56] T. Simon (2015). Positive stable densities and the bell-shape, Proc. Amer. Math. Soc. 143 No 2, 885895.

[57] B. Stankovič (1970). On the function of E.M. Wright. Publ. de lInstitut Mathématique, Beograd, Nouvelle Sér. 10, 113-124.

[58] F.G. Tricomi (1959). Funzioni Speciali, Gheroni, Torino.

[59] A. Wiman (1905a). Über den Fundamentalsatz der Theorie der Funkntionen $E_{\alpha}(x)$. Acta Math. 29, 191-201.

[60] A. Wiman (1905b). Über die Nullstellen der Funkntionen $E_{\alpha}(x)$. Acta Math. 29, 217-234.

[61] R. Wong and Y.-Q. Zhao (1999a). Smoothing of Stokes' discontinuity for the generalized Bessel function. Proc. R. Soc. London A455, 1381-1400.

[62] R. Wong and Y.-Q. Zhao (1999b). Smoothing of Stokes' discontinuity for the generalized Bessel function II, Proc. R. Soc. London A 455, 3065-3084.

[63] E.M. Wright (1933). On the coefficients of power series having exponential singularities. Journal London Math. Soc., 8, 71-79.

[64] E.M. Wright (1935). The asymptotic expansion of the generalized Bessel function. Proc. London Math. Soc. (Ser. II) 38, 257-270.

[65] E.M. Wright (1940). The generalized Bessel function of order greater than one. Quart. J. Math., Oxford Ser. $11,36-48$. 